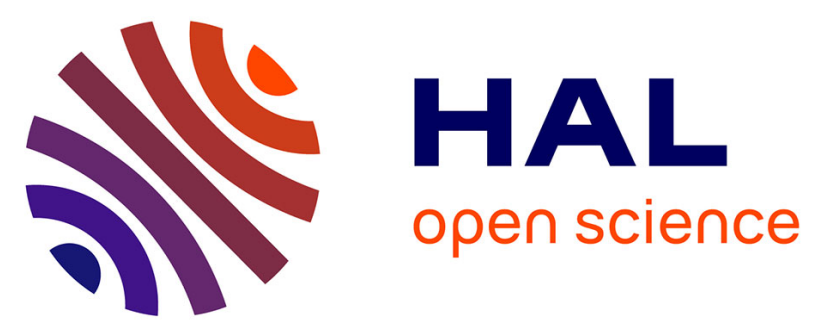

\title{
L\&apos;utilisation de 1\&apos;automobile par les ménages dans les territoires peu denses: analyse croisée par les enquêtes sur la mobilité et le Recensement de la population
}

\author{
Jean Paul Hubert, Pierre Pistre, Jean Loup Madre
}

\section{To cite this version:}

Jean Paul Hubert, Pierre Pistre, Jean Loup Madre. L\&apos;utilisation de 1\&apos;automobile par les ménages dans les territoires peu denses: analyse croisée par les enquêtes sur la mobilité et le Recensement de la population. Economie et Statistique / Economics and Statistics, 2016, 483-484485, pp 179-203. 10.3406/estat.2016.10678 . hal-01313813v2

\author{
HAL Id: hal-01313813 \\ https://hal.science/hal-01313813v2
}

Submitted on 29 Jan 2021

HAL is a multi-disciplinary open access archive for the deposit and dissemination of scientific research documents, whether they are published or not. The documents may come from teaching and research institutions in France or abroad, or from public or private research centers.
L'archive ouverte pluridisciplinaire HAL, est destinée au dépôt et à la diffusion de documents scientifiques de niveau recherche, publiés ou non, émanant des établissements d'enseignement et de recherche français ou étrangers, des laboratoires publics ou privés. 


\title{
L'utilisation de l'automobile par les ménages dans les territoires peu denses : analyse croisée par les enquêtes sur la mobilité et le Recensement de la population
}

\author{
Jean-Paul Hubert *, Pierre Pistre ** et Jean-Loup Madre ***
}

Rappel :

Les jugements et opinions exprimés par les auteurs n'engagent qu'eux mêmes, et non les institutions auxquelles ils appartiennent, ni a fortiori l'Insee.
Après des décennies ininterrompues de croissance, les distances parcourues en automobile par les ménages ont plafonné puis diminué, d'abord dans les grandes agglomérations, puis progressivement pendant les années 2000, dans des espaces de moins en moins denses. Or bien des explications données à ce phénomène dans le contexte urbain dense ne s'appliquent pas à des territoires où la mobilité dépend essentiellement de l'automobile. L'objectif de cet article est de mieux comprendre comment l'utilisation de l'automobile par les ménages s'est stabilisée puis a diminué dans les territoires peu denses, en combinant les sources statistiques sur la mobilité (Parc Auto et ENTD) et le Recensement de la population.

Cet ensemble de sources fait comprendre plusieurs dynamiques parallèles dans un contexte économique peu favorable. Tandis qu'une partie vieillissante des ménages continue à s'équiper en automobile et à rouler davantage, ceux qui disposent déjà de plusieurs véhicules réduisent les kilométrages parcourus en recentrant leurs activités plus près de chez eux et en reportant une partie de leur mobilité à longue distance sur d'autres modes de transport. En outre, les ménages comprenant un ou plusieurs actifs occupés, généralement multi-équipés, affectent de plus en plus leurs véhicules aux navettes domicile-travail, l'accès à l'emploi en dépendant.

Codes JEL : R23.

Mots clés : espaces peu denses, équipement automobile, utilisation automobile, domicile-travail.

* : Université Paris Est, IFSTTAR AME DEST, jean-paul.hubert@ifsttar.fr

** : Université Paris Est, IFSTTAR AME DEST, associé à l'UMR8504 - Géographie-cités, pierre.pistre@parisgeo.cnrs.fr

${ }^{* * \star}$ : Université Paris Est, IFSTTAR AME DEST, jean-loup.madre@ifsttar.fr

Cette étude fait partie d'un projet financé par le Ministère de l'Environnement, de l'Énergie et de la Mer (MEEM) dans le cadre du PREDIT 4 (2012-2016) intitulé La face cachée de la dynamique de la mobilité : rôle des zones peu denses dans l'évolution à long terme des territoires et de la mobilité (FACADYMO). Ce projet a pour objectif d'utiliser de manière plus approfondie les données de la statistique publique pour étudier la mobilité hors des grandes agglomérations.

Elle a aussi bénéficié d'une aide de l'État gérée par l'Agence nationale de la recherche au titre du programme Investissements d'avenir portant la référence ANR-10-EQPX-17 (Centre d'accès sécurisé aux données - CASD). L'utilisation du CASD pour cet article concerne, grâce à la levée du secret statistique sur le code commune des enquêtés de l'ENTD 2008, la possibilité de réaffecter ses données dans des typologies spatiales autres que celles proposées par l'Insee.

Nous remercions enfin la division Méthodes et traitements des recensements pour leur aide dans l'interprétation des données domicile-travail, ainsi que les relecteurs de la revue pour leurs avis constructifs. 
D epuis les années 1970 au moins, le kilométrage automobile ${ }^{1}$ par ménage a beaucoup crû grâce à l'augmentation du nombre de véhicules à disposition, alors que l'utilisation moyenne d'un véhicule pouvait au contraire baisser sur d'assez longues périodes (Join-Lambert et Thélot, 1981). Toutefois, d'après la Commission des Comptes des Transports de la Nation, la croissance des distances totales parcourues en automobile est stoppée depuis le milieu des années 2000 à l'échelle du pays (CCTN, 2015)², et la circulation totale plafonne comme dans la plupart des pays industrialisés (BITRE, 2011). Par comparaison avec les années 1990, on constate à partir de 2006 que l'utilisation de l'automobile par les résidents des grandes villes diminue, d'après les enquêtes ménages déplacements qui décrivent tous les 10 à 15 ans la mobilité à l'échelle d'un territoire urbain (De Solere, 2012). La comparaison entre les deux dernières enquêtes nationales sur les transports ${ }^{3}$ montre cependant que si le nombre de déplacements quotidiens est plus faible en 2008 qu'en 1994 dans les grandes agglomérations ${ }^{4}$, il est légèrement supérieur dans les territoires de faible densité corrélativement à un plus grand usage de l'automobile (Hubert, 2009). Mais l'usage de l'automobile n'a pas évolué de façon monotone pendant cette période. Utilisant le panel Parc Auto ${ }^{5}$, Grimal et al. (2013) montrent en effet que les kilométrages parcourus par adulte se stabilisent aussi dans l'espace peu dense à partir du début des années 2000, comme dans les espaces plus denses, mais à un niveau supérieur. Ce phénomène appelé "peak travel » (Goodwin, 2012) ou " plafonnement de la circulation » se traduit dans les villes par une baisse des kilométrages parcourus par les ménages. Il pourrait donc en être de même dans l'espace peu dense.

\section{Les ménages réduisent leur utilisation de l'automobile, mais les explications données pour le contexte urbain s'appliquent mal aux espaces peu denses}

$\mathbf{P}$ ourtant la plupart des explications à la saturation de la mobilité automobile dans les zones denses s'appliquent peu ou mal aux territoires à faible densité. La congestion, qui réduit l'efficacité de la voiture, et l'amélioration des transports en commun sont typiques du contexte des grandes villes. Ailleurs, l'offre de transports en commun demeure faible et difficile à financer (Paul-Dubois-Taine, 2012 ; Raoul et Casteigts, 2011). Elle est principalement destinée aux scolaires et l'utilisation des réseaux de transports régionaux requiert généralement la voiture pour accéder aux gares. Les circulations y restent majoritairement fluides car les vitesses pratiquées en voiture sont plus élevées lorsque la densité est plus faible (Hubert et Delisle, 2010). L'augmentation des distances à franchir y a donc moins d'impact sur le temps de transport. Les ménages y sont toutefois plus sensibles au prix des carburants (Orfeuil, 2013), qui ont flambé en 2008 et 2011 mais ont retrouvé fin 2015 un niveau comparable à ceux de 2005-2006 ou de 2009-2010 ${ }^{6}$.

D'autres explications mettent en avant des facteurs démographiques: l'équipement en automobile des ménages les plus jeunes et les plus mobiles a tendance à diminuer globalement (Démoli, 2015). Cette génération remplace donc progressivement une génération plus motorisée et joue un rôle essentiel dans le « plafonnement de la circulation » (Kuhnimhof et al., 2011). Dans le même temps, l'équipement des ménages plus âgés progresse, en taux et en nombre, en raison du remplacement de générations qui avaient eu peu accès à l'automobile par des générations plus nombreuses qui ont conduit toute leur vie (Metz, 2012 ; Madre et Bussière, 2002). Mais ces facteurs semblent avoir moins de portée dans les espaces peu denses. Les ménages jeunes et actifs ont généralement besoin de disposer d'une ou deux automobiles pour aller travailler et, selon différents indicateurs, ce besoin se renforce: les navettes domiciletravail s’allongent régulièrement (François, 2010)

\footnotetext{
1. Dans l'article, les termes " voiture », " automobile » ou " véhicule " désignent indifféremment les véhicules à disposition des ménages, généralement de type "voiture particulière ", parfois " véhicule utilitaire léger ", sauf lorsqu'il s'agit du mode de transport selon le RP, qui peut inclure un usage, rare, de camions. 2. Ce bilan est établi à partir des comptages de la circulation sur le réseau national et des consommations de carburants sur la base de clés de répartition données par des enquêtes de structure et un fichier du parc roulant. Pour les véhicules des ménages métropolitains, ce sont les enquêtes Panel carburant de Worldpanel Parc Au de Worldpanel, Parc Auto de la Sofres et l'enquête nationale Transports et déplacements 2008 (SOeS, Insee, Inrets).

3. Ces deux enquêtes se sont déroulées dans l'intervalle d'une année en 1993-94 et 2007-08 en France métropolitaine. Nous les désignons par leurs initiales et l'année la plus récente : ENT (enquête nationale sur les transports) 1994 et ENTD (enquête nationale sur les transports et déplacements) 2008.

4. La notion d'agglomération est ici appréhendée à partir des unités urbaines de l'Insee: des communes ou ensembles de communes "présentant une zone de bâti continu qui compte au moins 2000 habitants. ,

5. Pour une présentation succincte des données du panel Parc Auto, et de leur correspondance avec les données antérieures de l'enquête de conjoncture auprès des ménages (Ecam), voir Collet et al., 2012, p. 126.

6. Source Insee, indice des prix à la consommation : Carburants et lubrifiants (nomenclature COICOP : 07.2.2).
} 
et l'attraction des métropoles s'exerce toujours plus loin au sein des espaces peu denses (Floch et Levy 2011), d'autant plus que l'emploi local est affecté par le déclin économique des petites villes isolées (Davezies, 2012). La baisse d'utilisation de l'automobile dans l'espace peu dense n'est donc pas un simple prolongement de ce qui se produit dans l'espace dense, sauf peut-être dans les zones les plus proches de grandes villes. Il faut chercher d'autres explications dans l'évolution des comportements d'usage de l'automobile selon les contextes sociaux et spatiaux. Par exemple : une réorganisation de la mobilité quotidienne autour du domicile, comme le montrent Berger et al. (2015) pour les ménages périurbains franciliens; le développement dans les communes rurales du travail à domicile, sous statut d'auto-entrepreneur et en configuration de télétravail (Ortar, 2009); une moindre utilisation de la voiture pour les voyages à longue distance, mise en évidence par Grimal (2010) entre 1994 et 2008, mais ayant concerné en priorité les voyages à motif professionnel; ou encore une dévolution des véhicules aux navettes domicile-travail, mise en exergue par Le Breton (2008) pour les ménages les moins aisés cherchant à économiser le carburant et le véhicule.

L'objectif de cet article est double : d'une part établir que les kilométrages moyens parcourus par les ménages résidant dans l'espace peu dense diminuent depuis le début des années 2000, alors que l'automobile est essentielle à leur mobilité et, d'autre part, expliciter à partir de différentes sources statistiques les mécanismes sous-jacents à ce processus.

L'analyse comporte cinq parties. La première examine la dépendance à l'automobile et l'évolution des kilométrages des ménages dans l'espace peu dense en comparaison avec les agglomérations. La deuxième présente le cadre géographique de l'étude, l'espace peu dense, et l'outil typologique construit pour en appréhender la diversité, grâce au $R P$ ou aux enquêtes auprès des ménages ${ }^{7}$. La troisième partie présente, en les différenciant par type de ménages et d'espace peu dense, deux indicateurs clés de l'usage de l'automobile : l'équipement des ménages en automobile, qui croît mais tend à saturer, et le kilométrage annuel moyen de leurs véhicules, en baisse constante depuis le milieu des années 1990, après une hausse d'au moins une dizaine d'années. Cette partie conclut sur le fait que, depuis 2008 au moins, la moindre utilisation des véhicules n'est plus compensée par l'augmentation de la motorisation et entraîne une baisse pratiquement équivalente du kilométrage moyen par ménage. La quatrième partie examine l'évolution des distances domicile-travail et de la part modale de l'automobile pour aller travailler, par type d'espace et par classe de distance, afin d'analyser l'impact de l'allongement des navettes domicile-travail sur la circulation, selon le type d'espace. La cinquième partie est plus exploratoire : elle vise à repérer des changements de comportements dans l'usage des véhicules, en faisant l'hypothèse que l'utilisation de la voiture est de plus en plus centrée sur le domicile-travail au détriment des motifs non obligés. Reprenant les différents résultats de l'article, la conclusion avance une conjecture. Des éléments plus techniques sur la typologie géographique et sur les traitements réalisés à partir des fichiers détail anonymisés LOGEMT (ménages) et MOBPRO (actifs occupés) pour produire des séries annualisées sont donnés en annexe.

\section{Quelles statistiques mobiliser pour analyser l'évolution des usages de l'automobile?}

Depuis l'enquête nationale Transports et déplacements de 2007-08, il n'existe pas de source statistique donnant une vision globale des utilisations de l'automobile par les ménages vivant dans l'espace peu dense et de leur motorisation ; la dernière enquête aussi détaillée remonte à 1993-94. Pour analyser les évolutions depuis le début des années 2000, nous devons donc combiner les sources nationales sur la mobilité et le recensement.

L'interrogation du panel Parc Auto est annuelle mais l'échantillon est limité (ex : 2409 ménages en 2011 qui résident hors des unités urbaines de 10000 habitants et plus). Il permet d'estimer approximativement la part du kilométrage annuel réalisé pour aller au travail et depuis 2009, pour partir en weekend ou en vacances. Les enquêtes nationales sur les transports (ENT 1994 et ENTD 2008) reposent sur un échantillon plus large (par exemple, en 2008, 8679 ménages ${ }^{8}$ résidant hors des unités urbaines de 10000 habitants et plus) et donnent une image détaillée de la mobilité.

\footnotetext{
7. La diversité de l'espace peu dense est difficile à reconstituer avec les catégories de l'Insee construites pour suivre la dynamique de l'urbanisation. Même si la typologie 2010 des Aires Urbaines distingue trois tailles de pôles et peut être croisée avec les tranches d'unités urbaines 2010, de grandes classes de communes restent mal différenciées. Par exemple: les couronnes périurbaines très étendues des principales métropoles ou encore les communes multipolarisées (33,5\% des communes de l'espace peu dense, seulement partageables en $11,8 \%$ de communes multipolarisées des grandes aires urbaines et $21,7 \%$ d'autres multipolarisées).

8. Compte tenu d'un suréchantillonnage destiné à mieux observer les zones peu denses.
} 
Le Recensement de la population permet d'examiner finement deux déterminants de l'usage de l'automobile : l'équipement des ménages et la mobilité domicile-travail. Cependant, il ne donne pas d'indications sur les kilométrages parcourus, contrairement aux sources panel Parc Auto et ENT. Il a en outre un avantage particulier pour l'étude de l'espace peu dense car depuis 2004, les communes de moins de 10000 habitants sont intégralement recensées tous les cinq ans dans une rotation annuelle entre 5 groupes de communes, constitués pour produire des échantillons équilibrés au niveau régional (Insee, 2005). En retirant les communes intégrées à des agglomérations importantes pour se limiter à l'espace peu dense, cet équilibre peut être altéré mais les Enquêtes annuelles de recensement (EAR) offrent néanmoins la possibilité de construire des séries annuelles à partir d'échantillons bien fournis (Brutel, 2014a ; Brutel, 2015b ; Madre et al., 2015). Si la plupart des grands domaines d'étude statistique, comme la production économique ou la démographie naturelle, disposent de sources de données robustes et au minimum annuelles (comptabilité nationale, état civil, etc.), le champ des transports et des mobilités manque de données permettant un suivi régulier et de court terme des tendances à des échelles fines. Cette exploitation par EAR est donc particulièrement intéressante : elle permet de conforter l'information du panel Parc Auto sur la motorisation et la mobilité domicile-travail, et de la décliner selon les territoires ou les types de ménage, complétant ainsi les informations tirées des ENT 1994 et ENTD 2008. Ce traitement des données du recensement dans les communes de moins de 10000 habitants apporte une information plus continue sur une période marquée par la stagnation économique.

\section{Les territoires peu denses dépendent de l'automobile, pourtant son utilisation par les ménages baisse après 2001}

$\mathbf{E}$ n 2008, selon l'enquête nationale ENTD, plus des trois quarts des déplacements de semaine ou de week-end des ménages résidant dans les espaces peu denses sont effectués en voiture, soit comme conducteur, soit comme passager (cf. tableau 1). Leurs véhicules roulent

Tableau 1

Usage de l'automobile pour la mobilité quotidienne en France métropolitaine selon les tranches d'unités urbaines 2010

\begin{tabular}{|c|c|c|c|c|c|c|c|c|c|}
\hline & & \multirow{2}{*}{\multicolumn{2}{|c|}{$\begin{array}{l}\text { Part des actifs } \\
\text { allant au travail } \\
\text { régulièrement } \\
\text { en voiture (1) }\end{array}$}} & \multicolumn{4}{|c|}{$\begin{array}{l}\text { Part des déplacements } \\
\text { en automobile (2) }\end{array}$} & \multirow{2}{*}{\multicolumn{2}{|c|}{$\begin{array}{c}\text { Utilisation } \\
\text { moyenne par } \\
\text { semaine d'une } \\
\text { automobile (3) }\end{array}$}} \\
\hline \multirow{2}{*}{\multicolumn{2}{|c|}{$\begin{array}{l}\text { Catégories de communes } \\
\text { (d'après les tranches } \\
\text { d'unités urbaines 2010) }\end{array}$}} & & & \multicolumn{2}{|c|}{$\begin{array}{c}\text { Lundi } \\
\text { à vendredi }\end{array}$} & \multicolumn{2}{|c|}{$\begin{array}{l}\text { Samedi } \\
\text { et dimanche }\end{array}$} & & \\
\hline & & $\begin{array}{l}\text { Actif } \\
(\%)\end{array}$ & $\begin{array}{l}\text { dont sans } \\
\text { autre } \\
\text { choix (\%) }\end{array}$ & $\begin{array}{l}\text { Déplct } \\
(\%)\end{array}$ & $\begin{array}{l}\mathrm{Km} \\
(\%)\end{array}$ & $\begin{array}{l}\text { Déplct } \\
(\%)\end{array}$ & $\begin{array}{l}\mathrm{Km} \\
(\%)\end{array}$ & $\begin{array}{l}\text { Nombre } \\
\text { de } \\
\text { déplct }\end{array}$ & $\mathrm{Km}$ \\
\hline \multirow{2}{*}{ Espace peu dense } & Hors des UU & 90 & 82 & 81 & 91 & 80 & 94 & 17,1 & 231 \\
\hline & UU de 2000 à 9999 habs. & 84 & 71 & 70 & 89 & 74 & 93 & 17,6 & 211 \\
\hline Petites agglomérations & UU de 10000 à 49999 habs. & 75 & 59 & 73 & 90 & 76 & 93 & 19,3 & 213 \\
\hline Moyennes agglomérations & UU de 50000 à 199999 habs. & 77 & 51 & 66 & 84 & 68 & 91 & 19,9 & 199 \\
\hline Grandes agglomérations & UU de 200000 habs. et plus & 71 & 45 & 61 & 82 & 70 & 92 & 17,6 & 169 \\
\hline Agglomération de Paris & UU de Paris & 38 & 29 & 39 & 56 & 51 & 77 & 14,1 & 148 \\
\hline \multicolumn{2}{|l|}{ France métropolitaine } & 72 & 60 & 65 & 83 & 70 & 91 & 17,5 & 198 \\
\hline
\end{tabular}

Lecture : en France métropolitaine, $72 \%$ des actifs ayant un lieu de travail fixe déclarent y aller régulièrement en voiture, comme conducteur ou passager. Parmi ceux-ci $60 \%$ déclarent ne pas avoir d'autre choix de transport. Un jour avec déplacements, en semaine, $65 \%$ des déplacements à moins de $80 \mathrm{~km}$ du domicile sont réalisés en voiture, représentant $83 \%$ des kilomètres parcourus ; respectivement $70 \%$ et $91 \%$ un samedi ou un dimanche. Pendant 7 jours, une voiture sert à réaliser 17,5 déplacements de moins de $100 \mathrm{~km}$ qui totalisent $198 \mathrm{~km}$ au compteur.

Champ : (1) : actifs travaillant dans un lieu de travail fixe à moins de $100 \mathrm{~km}$ du domicile et partant au travail de leur domicile ; (2) : déplacements quotidiens à moins de $80 \mathrm{~km}$ à vol d'oiseau du domicile de résidents métropolitains de 6 ans et plus ; (3) voitures ou véhicules utilitaires légers en état de marche à disposition des ménages et déplacements de moins de $100 \mathrm{~km}$ au compteur (approximativement $80 \mathrm{~km}$ à vol d'oiseau).

Source : Service de l'observation et des statistiques du Ministère de l'Environnement, de l'Énergie et de la Mer (SOeS), Insee, Inrets, enquête nationale Transports et Déplacements 2008. 
davantage. Plus de 8 actifs sur 10 ayant un lieu de travail fixe hors du domicile vont à leur travail uniquement en voiture et déclarent très majoritairement ne pas avoir d'autre choix.

Nous pouvons vérifier que les espaces peu denses ne sont pas affectés par la baisse de motorisation des jeunes ménages observée dans les villes. Hors des unités urbaines de 10000 habitants et plus, les ménages dont la personne de référence a moins de 40 ans restent au même niveau très élevé de motorisation entre 2006 et 2011, contrairement à ceux résidant dans une unité urbaine plus importante (cf. figure I). La première voiture continue d'en appeler une deuxième chez ces ménages jeunes, dans les espaces peu denses mais aussi dans les petites unités urbaines (10 000 à 50000 habitants) où le taux de motorisation par adulte des ménages équipés augmente entre 2006 et 2011, alors que ce taux stagne ou diminue légèrement dans les agglomérations plus grandes.

De manière générale, l'équipement en automobile continue de progresser pour l'ensemble des ménages de l'espace peu dense : en 2011, 90,3 \% des ménages disposaient d'au moins une voiture contre 89,0 \% en 2006. En comparaison, la motorisation des ménages stagne dans les espaces denses, voire régresse : 76,2\% en 2011 (- 0,2 point par rapport à 2006). L'usage d'automobiles pour aller au travail a aussi continué à s'intensifier dans l'espace peu dense, passant de 81,4 \% en 2006 à 82,9 \% en 2011 alors qu'il baissait dans le reste du territoire métropolitain, de 63,9 \% à 62,1 \% (données $R P$ ).

Le panel Parc Auto permet de suivre l'évolution des kilomètres parcourus annuellement par ménage, dans l'espace peu dense et dans

\section{Figure I}

Motorisation des ménages et motorisation par adulte des ménages équipés, lorsque la personne de référence a moins de $\mathbf{4 0}$ ans, selon la taille de l'unité urbaine de résidence.

Évolution 2006-2011

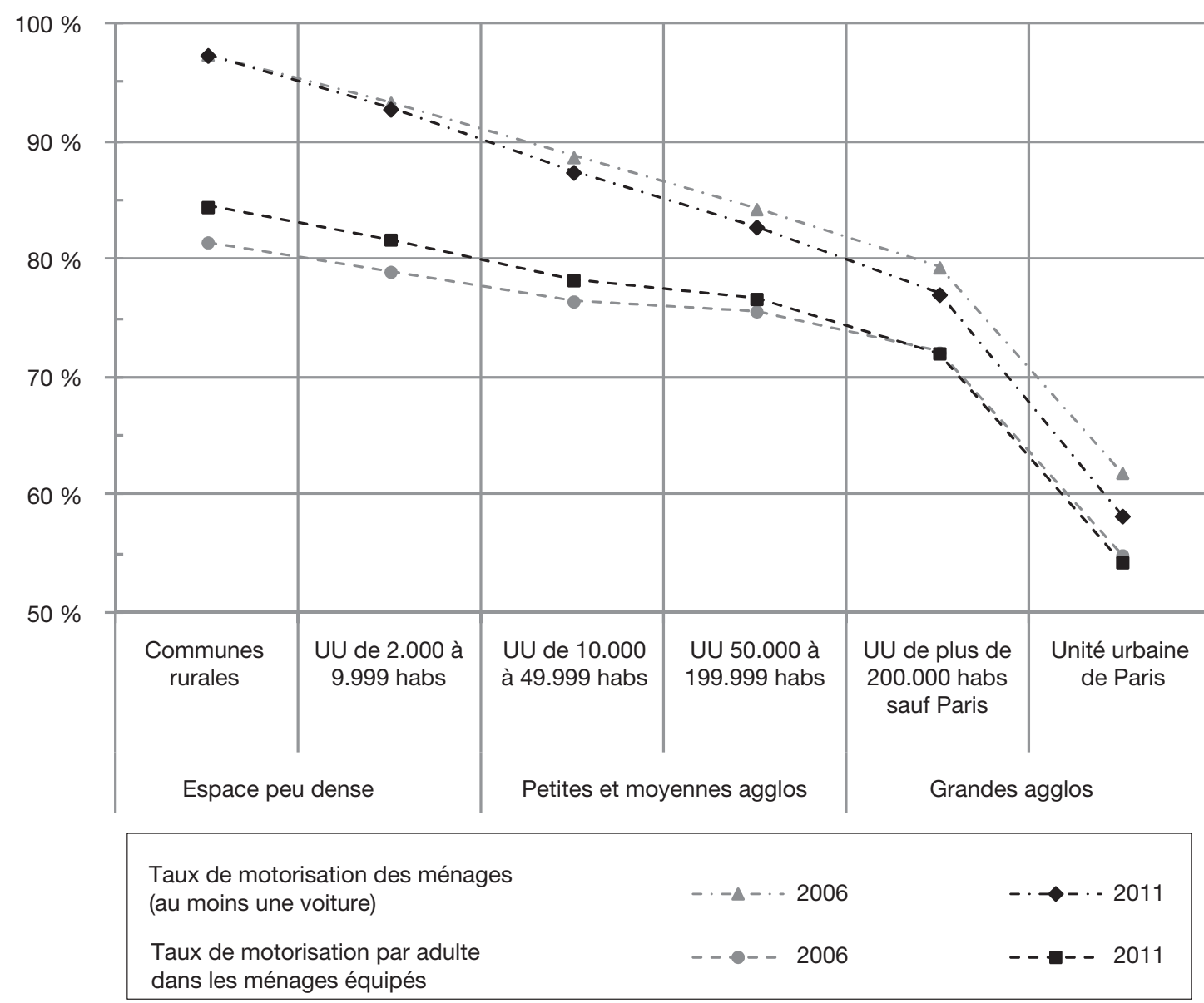

Champ : ménages qui résident en France métropolitaine et dont le chef a moins de 40 ans.

Source : Insee, Recensement de la population 2006 et 2011 (exploitation principale). 
les agglomérations. Dans l'agglomération parisienne, ce kilométrage se stabilise et commence à baisser dès le début des années 1990. Le même phénomène se produit plus tardivement, et à un niveau chaque fois supérieur à mesure que l'on descend dans l'échelle des tailles d'agglomération, jusqu'à l'espace peu dense à partir de 2001, au niveau de 20000 km/an environ (cf. figure II) ${ }^{9}$. Les points d'observation des enquêtes nationales sur les transports sont bien situés de part et d'autre de la date pivot. Dans sa trajectoire à la baisse, le point 2008 n'est pas encore au niveau de 1994, ce qui explique l'apparente progression de la mobilité automobile entre l'ENT 1994 et l'ENTD 2008 mentionnée en introduction.

9. L'espace peu dense désigne tout au long de cet article les communes qui n'appartiennent pas aux unités urbaines de 10000 habitants et plus : c'est-à-dire les communes non agglomérées de moins de 2000 habitants, ainsi que les communes des unités urbaines de 2000 à 9999 habitants.

\section{Figure II \\ Évolution du nombre annuel moyen de kilomètres parcourus par ménage selon la catégorie de commune de résidence en France métropolitaine (panel ParcAuto)}

En km

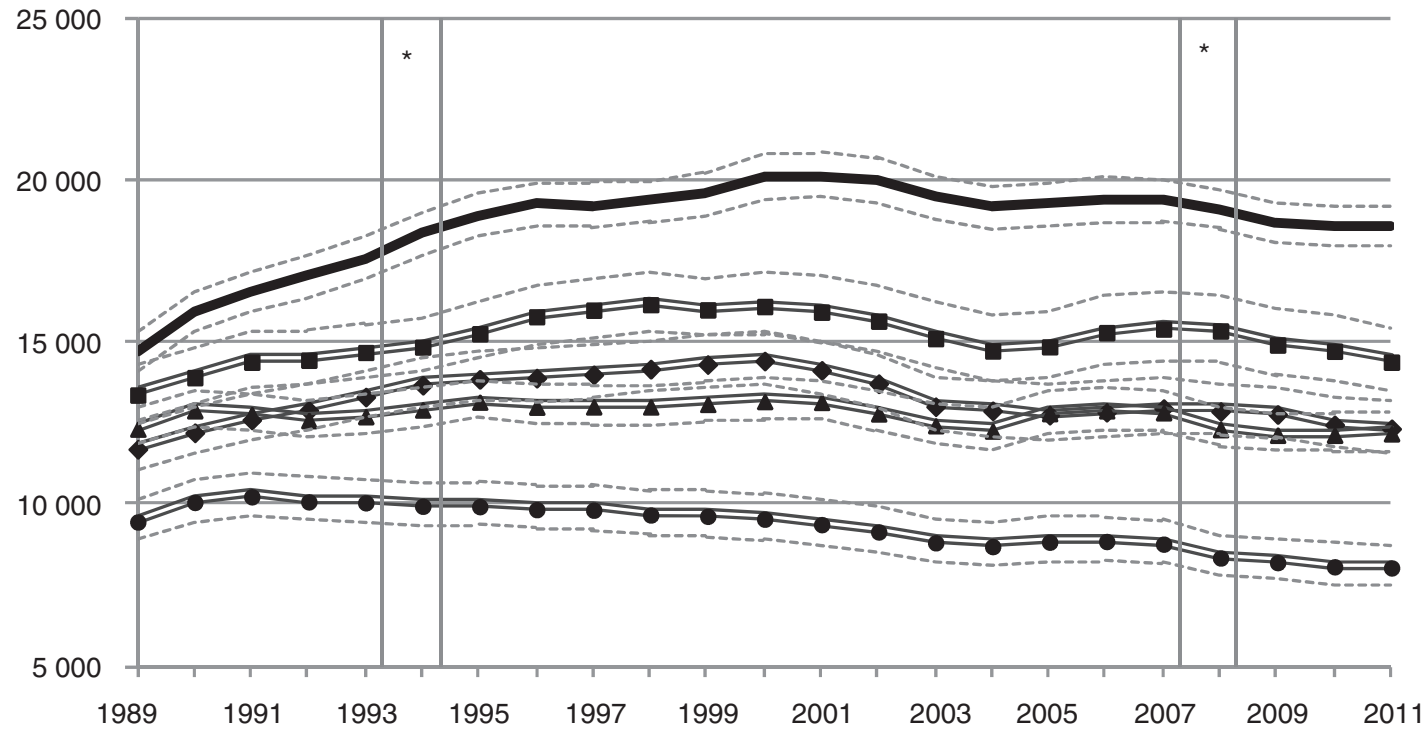

*Périodes de réalisation des deux dernières enquêtes nationales transport

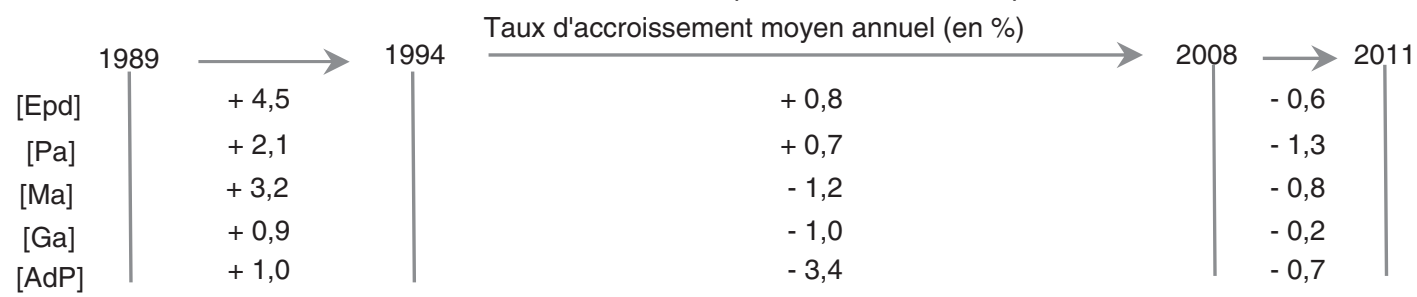

\begin{tabular}{|c|c|c|}
\hline \multicolumn{3}{|c|}{ Catégories de communes (d'après les tranches d'Unités Urbaines 2010) } \\
\hline $\begin{array}{l}\text { [Epd] Espace peu } \\
\text { dense (hors des UU de } \\
10.000 \text { habs et plus) }\end{array}$ & $\begin{array}{l}\text { [Ma] Moyennes } \\
\text { agglomérations (UU de } \\
50.000 \text { à } 199.999 \text { habs) }\end{array}$ & $\begin{array}{c}\Longrightarrow[\text { AdP }] \text { Agglomération } \\
\text { de Paris }\end{array}$ \\
\hline $\begin{aligned} \longrightarrow & {[\mathrm{Pa}] \text { Petites } } \\
& \text { agglomérations (UU de } \\
& 10.000 \text { à } 49.999 \text { habs) }\end{aligned}$ & $\begin{array}{l}\square \text { [Ga] Grandes } \\
\text { agglomérations (UU de } \\
200.000 \text { habs et plus) }\end{array}$ & $\begin{array}{l}\text {------ Intervalles de } \\
\text { confiance }\end{array}$ \\
\hline
\end{tabular}

Lecture : données annuelles lissées par moyennes mobiles sur 3 ans.

Champ : ménages qui résident en France métropolitaine.

Source : Insee, ECAM (1987-1994) et TNS Sofres, panel Parc Auto (1994-2013). 


\section{Différencier l'espace peu dense dans la perspective de la mobilité}

$\mathbf{I}^{1}$

1 y a bien des façons de définir les territoires peu denses qui peuvent les faire apparaître plus ou moins homogènes. Dans le cadre de la prospective Territoires 2040 initiée par la Datar, le groupe de travail sur l'avenir des «espaces de la faible densité » (Barthe et Milian, 2012) a adopté une définition ne conservant que les communes de moins de 30 habitants par $\mathrm{km}^{2}$, soit environ $48 \%$ de la superficie du territoire national et 5,3 millions d'habitants. Une autre approche est utilisée par Hilal et al. (2012) dans leur définition extensive et plurifactorielle des campagnes françaises, composées des communes hors des pôles urbains de plus de 10000 emplois. Nous adoptons également une définition élargie de l'espace peu dense mais dans la perspective de la mobilité. C'est l'espace où la voiture permet de se déplacer rapidement, c'est-à-dire jusqu'à la limite des espaces densément bâtis que nous assimilons aux agglomérations de 10000 habitants et plus. Nous retenons ainsi 8,9 millions de ménages ordinaires, soit 21,4 millions d'habitants disposant de 1,3 millions de voitures ( $R P$ 2008) et vivant sur environ $89 \%$ du territoire national.

Pour différencier cet espace peu dense selon un critère non régional et utilisable dans une base de données produite par une enquête nationale, nous distinguons trois types de configurations spatiales sur la base d'une classification des communes construites à partir des densités de population locale et de voisinage (cf. annexe 1). Le croisement de ces deux densités permet en effet de distinguer trois figures typiques de territoires qui composent l'espace visé par l'étude (cf. carte) : les espaces de la très faible densité, les petits pôles et les espaces à dominante périurbaine, lesquels sont encore déclinés en fonction du degré de densité de voisinage, lié principalement à la taille du pôle urbain dont ils dépendent.

Ces types sont désignés dans la suite à partir de regroupements de classes de densités croisées :

1 - « Espaces de très faible densité » : les campagnes les plus isolées (étiquetés A1a);

2 - « Petits pôles » : bourgs et petites villes isolées, généralement en fort déclin $(\mathrm{A} 1 \mathrm{~b}+\mathrm{B} 1)$;

3 - « Espaces à dominante périurbaine » : (i) « les moins denses », souvent à la marge des couronnes périurbaines $(\mathrm{A} 1 \mathrm{c}+\mathrm{A} 1 \mathrm{~d})$;

(ii) « de densité intermédiaire », souvent autour d'un pôle moyen ou à distance moyenne d'un pôle important (A2+B2) ;

(iii) « les plus denses », territoires métropolisés autour des plus grandes agglomérations (A3+B3).

L'espace peu dense est déficitaire en emploi. Au $R P$ 2011, il comptait seulement 6210000 emplois pour 9401000 actifs occupés, soit deux emplois pour trois actifs résidents. Au bilan : $44,4 \%$ des actifs (4,1 millions) travaillent dans les unités urbaines de 10000 habitants et plus, tandis que $16 \%$ des emplois (environ un million) sont occupés par des habitants venant des zones denses. Les territoires à dominante périurbaine sont structurellement plus déficitaires en emploi, puisque leur population a crû par l'installation de ménages dont un ou deux actifs travaillent dans l'espace dense. Seuls les petits pôles ont un bilan favorable (huit emplois pour sept actifs occupés), mais ceci cache une tendance à la baisse du nombre de résidents actifs occupés et du nombre d'emplois depuis 2006. Cet espace est hétérogène, tiraillé par les différences de dynamisme entre différents pôles urbains. Les territoires périurbains attenants aux grandes agglomérations renforcent leur base économique locale. Les autres espaces périurbains sont dans des situations de multipolarisation, en devenant davantage dépendants de pôles éloignés mais plus dynamiques que ceux de leur voisinage. Les petits pôles sont touchés de plein fouet par la crise. Quant aux territoires les moins denses, l'emploi lointain prend aussi le pas sur l'emploi local où les statuts d'indépendants non agricoles compensent pour partie la diminution du nombre des agriculteurs.

\section{Équipement automobile stabilisé et utilisation en baisse des véhicules}

T es indicateurs de motorisation peuvent être suivis annuellement par la série des $E A R^{10}$ (cf. encadré 1 et annexe 2). Elle montre

10. Les séries brutes des EAR pour les variables de motorisation montrent une grande régularité de tendance, c'est pourquoi elles sont préférées à une moyenne mobile sur 3 ans moins précise. L'effet de cycle de rotation paraît négligeable, sauf pour la classe peu nombreuse des ménages actifs sans emplois. 
que l'équipement progresse surtout pour les ménages d'inactifs, alors que du côté des actifs, la motorisation atteint certains seuils de saturation (cf. figure III).

Carte

Version agrégée de la typologie des communes françaises selon les densités de population locale et de voisinage

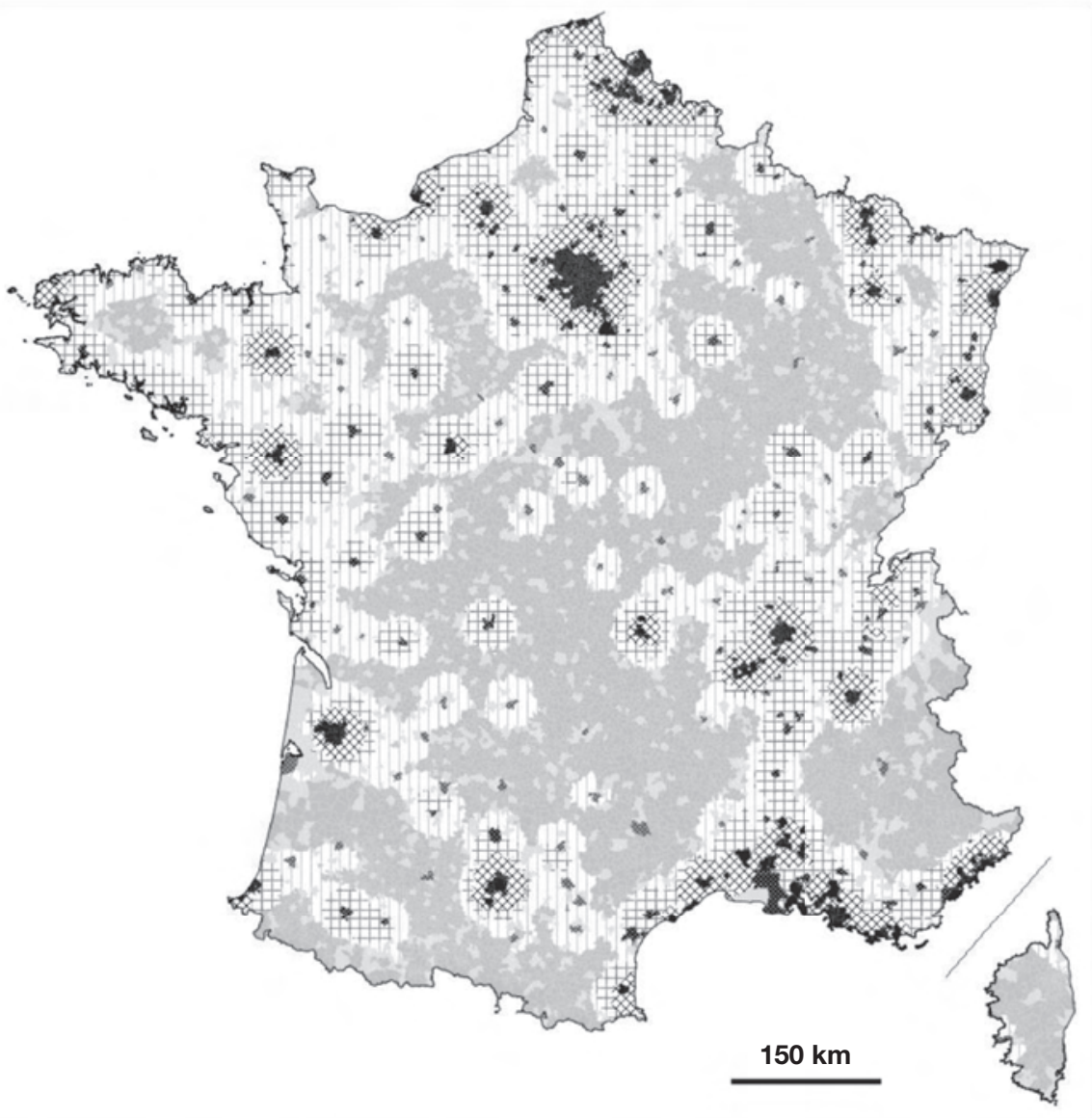

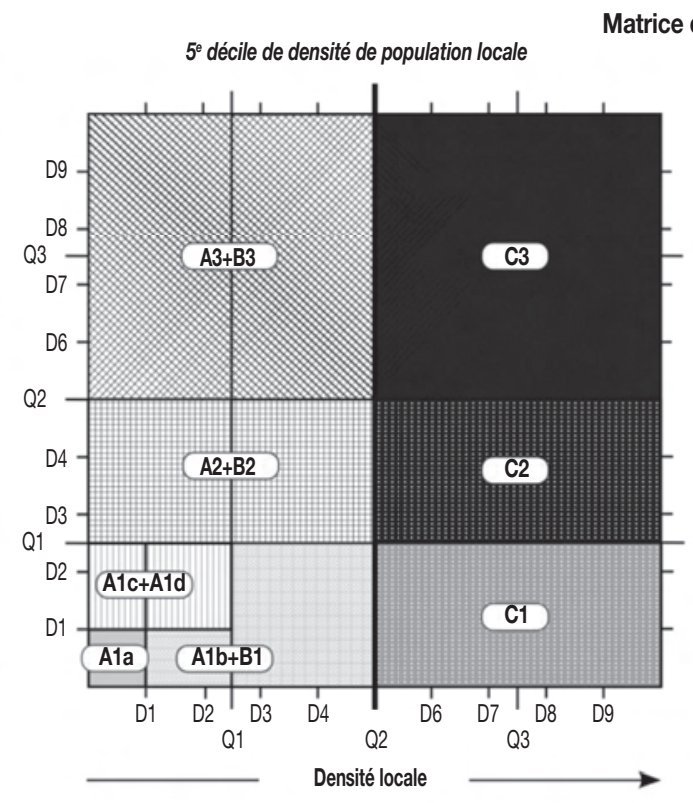

Déciles et quartiles de densité de cercles de $5 \mathrm{~km}$ de diamètre autour des chefs lieux communaux
Matrice de classification des communes françaises selon les densités de population locale et de voisinage

Densités locales des communes inférieures au $5^{e}$ décile

A1a Communes des espaces de très faible densité

A1b+B1 Communes des petits pôles

A1c+A1d Communes des espaces périurbains les moins denses

A2+B2 Communes des espaces périurbains de densité intermédiaire

A3+B3 Communes des espaces périurbains les plus denses

Densités locales des communes supérieures au $5^{\circ}$ décile

C1 Communes des pôles moyens avec un voisinage peu dense

Communes des pôles moyens avec un voisinage de densité intermédiaire

Communes des grands pôles avec un voisinage dense et plus

Source : Insee, Recensement de la population 2009 données carroyées - $1 \mathrm{~km}$ de côté) IGN, GEOFLA édition 2013 


\section{L'équipement des ménages proche de la saturation, sauf pour les plus âgés}

Les ménages biactifs sont quasiment intégralement motorisés. Chez les ménages équipés en automobile et ayant au moins un actif occupé, la motorisation par adulte a ralenti après 2006, avec une inflexion située juste au-dessus de $80 \%$.
Pour les inactifs les plus âgés, le remplacement des cohortes anciennes où les femmes ne conduisaient pas, par des cohortes où la plupart des adultes conduisent, fait augmenter rapidement le nombre de voitures des ménages. Pour les inactifs de moins de 75 ans, majoritairement issus des générations du baby-boom, la pratique usuelle et partagée de la conduite s’accompagne

Encadré 1

\section{SUIVRE ANNUELLEMENT CERTAINS INDICATEURS POUR LES COMMUNES DE MOINS DE 10000 HABITANTS}

Le nouveau dispositif de recensement a annualisé depuis 2004 le processus de collecte des données par cycle quinquennal. Les communes de moins de 10000 habitants ont conservé un recensement exhaustif, qui assure une base territoriale stable aux évolutions mesurées sur 5 ans. Pour ces communes, l'extraction des données d'une EAR à partir des fichiers détail ne pose pas de difficultés techniques, mais de légers déséquilibres entre les groupes peuvent biaiser les estimateurs (cf. annexe 2).

Les séries temporelles utilisées dans l'article sont ainsi de deux ordres :

- de manière conventionnelle, une partie des analyses repose sur la comparaison des résultats entre deux recensements généraux réalisés au cours des années 2000 - parfois complétés par les résultats au $R P$ 1999. Les $R P 2006$ (EAR 2004 à 2008) et $R P 2011$
(EAR 2009 à 2013) sont utilisés ; lors de la mise en ligne des données du RP 2011 (en 2014), ils étaient les premiers à permettre une comparaison directe des résultats entre deux recensements réalisés selon la nouvelle méthode :

- les séries originales par EAR sont exploitées sous deux formes afin de contrôler l'effet des groupes de rotation: (1) des séries annuelles brutes des variables en niveau lorsqu'elles présentent très peu d'irrégularités cycliques ou des séries lissées par moyennes mobiles sur 3 ans dans les représentations graphiques ; (2) des variations quinquennales (2004-2009, 2005-2010, ... , 2008-2013) qui permettent une analyse en évolution sur un même groupe de rotation, que l'on suppose représentatif de l'évolution sur l'ensemble de l'espace peu dense, sans pouvoir entrer dans le détail des types d'espace (cf. annexe 2).

Figure III

Évolution de la motorisation dans l'espace peu dense selon la composition des ménages en actifs et en inactifs

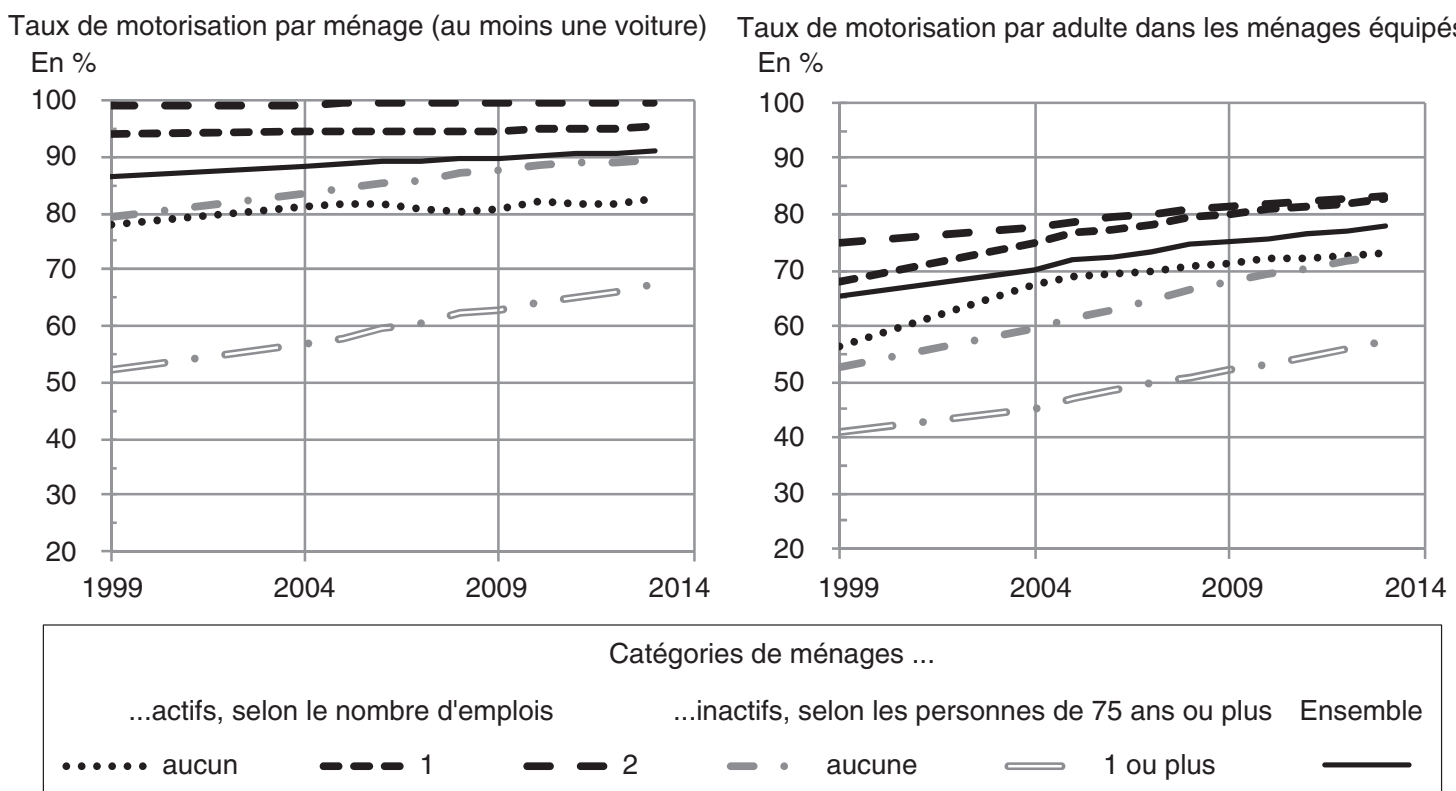

Champ : ménages qui résident dans les communes hors des unités urbaines de 10000 habitants et plus.

Source : Insee, Recensement de la population 1999 (exploitation complémentaire), 2006 et 2011 (exploitation principale). 
également de revenus en moyenne plus élevés qui facilitent l'équipement.

L'augmentation du nombre de ménages inactifs ou sans emploi est régulière : presque $2 \%$ par an depuis 2004 (cf. tableau 2). Le nombre de ménages avec actifs occupés a augmenté de $6,7 \%$ entre 2004 et 2009 mais seulement de 3,6 \% au cours de la période 2008-2013 qui s'inscrit presque entièrement dans une conjoncture peu favorable. L'accroissement du parc automobile résulte donc de plus en plus des acquisitions des ménages inactifs ou chômeurs.

\section{Après un palier vers la fin des années 90, des véhicules qui roulent de moins en moins}

D ans l'espace peu dense, une voiture parcourait en 2011 environ 12900 km par an, selon le panel Parc Auto ${ }^{11}$. Son kilométrage annuel était nettement plus élevé si elle appartenait à un ménage dont l'un au moins des membres travaille ${ }^{12}$. Le kilométrage annuel d'un véhicule diminue de plus de $1 \%$ par an depuis 1999 et la baisse est sensiblement plus forte chez les ménages sans actif occupé depuis 2006. La différence d'utilisation entre ménages avec ou sans actifs occupés s’est donc accrue. Néanmoins, tous les ménages montrent une même tendance à moins utiliser leurs voitures (cf. tableau 3). La série complète, qui

11. Rappelons qu'avec un nombre de véhicules par ménage de l'ordre de 1,4 cette valeur est bien cohérente avec les 18 à 20000 kilomètres parcourus annuellement par les ménages de la figure II.

12. En 2007-2008, l'écart de kilométrage entre la voiture d'un ménage avec actif occupé ou sans, est de $3922 \mathrm{~km}$ par an selon le panel Parc Auto et de $5078 \mathrm{~km}$ selon l'ENTD. Les enquêtes diffèrent mais les intervalles de confiance à $95 \%$ de ces écarts se recoupent. Les évolutions entre 1993-94 et 2007-08 sont assez concordantes : - $7 \%$ selon Parc Auto et $-4 \%$ selon les ENT 1994 et ENTD 2008, malgré les différences méthodologiques et compte tenu du fait que les points d'observation sont situés, l'un dans une phase de croissance, l'autre de décroissance.

\begin{tabular}{|c|c|c|c|c|c|c|}
\hline & \multicolumn{5}{|c|}{ Variations quinquennales par EAR (taux de croissance en \%) } \\
\hline & & 2004-2009 & $2005-2010$ & $2006-2011$ & $2007-2012$ & $2008-2013$ \\
\hline \multirow{3}{*}{ Nombre de ménages } & Actifs avec emploi(s) & $+6,7$ & $+6,2$ & $+5,6$ & $+4,6$ & $+3,6$ \\
\hline & Inactifs ou sans emploi & $+10,0$ & $+10,2$ & $+9,7$ & $+10,0$ & $+10,1$ \\
\hline & Ensemble & $+7,9$ & $+7,7$ & $+7,1$ & $+6,6$ & $+6,0$ \\
\hline \multirow{3}{*}{ Nombre de voitures } & Actifs avec emploi(s) & $+9,2$ & $+8,0$ & $+7,2$ & $+6,2$ & $+5,2$ \\
\hline & Inactifs ou sans emploi & $+21,6$ & $+21,3$ & $+19,4$ & $+19,0$ & $+18,2$ \\
\hline & Ensemble & $+12,2$ & $+11,2$ & $+10,2$ & $+9,4$ & $+8,5$ \\
\hline \multirow{3}{*}{$\begin{array}{l}\text { Nombre de ménages motorisés } \\
\text { (au moins une voiture) }\end{array}$} & Actifs avec emploi(s) & $+6,9$ & $+6,4$ & $+5,9$ & $+4,9$ & $+4,0$ \\
\hline & Inactifs ou sans emploi & $+16,3$ & $+16,5$ & $+15,0$ & $+15,1$ & $+14,7$ \\
\hline & Ensemble & $+9,8$ & $+9,5$ & $+8,7$ & $+8,1$ & $+7,3$ \\
\hline
\end{tabular}

Champ : ménages qui résident dans les communes hors des unités urbaines de 10000 habitants et plus. Source : Insee, Recensement de la population 2006 et 2011 (exploitation principale).

Tableau 3

Évolution du kilométrage annuel moyen d'un véhicule d'un ménage avec ou sans actif occupé dans l'espace peu dense (Parc Auto écrêté à $50000 \mathrm{~km}$ par an)

\begin{tabular}{|l|c|c|c|c|c|}
\hline & \multicolumn{3}{|c|}{ Kilométrage annuel en } & \multicolumn{2}{c|}{ Évolution sur 5 ans } \\
Véhicule à disposition d'un... & $\begin{array}{c}1999(\mathrm{~km}) \\
\text { (moy.1997-2001) }\end{array}$ & $\begin{array}{c}2006(\mathrm{~km}) \\
\text { (moy.2004-2008) }\end{array}$ & $\begin{array}{c}2011(\mathrm{~km})(\mathrm{moy} . \\
2009-2013)\end{array}$ & $\begin{array}{l}1999-2006(\%) \\
2006-2011(\%)\end{array}$ \\
\hline ...ménage sans actif occupé & 12538 & 11322 & 10100 & $-7,0$ & $-10,8$ \\
...ménage avec actif occupé & 16429 & 14915 & 14233 & $-6,7$ & $-4,6$ \\
Ensemble des véhicules & 14925 & 13605 & 12898 & $-6,4$ & $-5,2$ \\
\hline
\end{tabular}

Lecture : moyennes lissées et évolutions rapportées à une période de 5 ans.

Champ : ménages qui résident dans les communes hors des unités urbaines de 10000 habitants et plus.

Source : TNS Sofres, panel Parc Auto (1997-2013). 
commence en 1984, montre que cette phase de baisse fait suite à un palier entre 1994 et 2001, pendant lequel le kilométrage pour l'ensemble des véhicules s'est maintenu entre 14500 et $15000 \mathrm{~km} / \mathrm{an}$. Auparavant, on observe une phase de hausse pendant laquelle ce même kilométrage est passé de 12400 km/an en 1984-86 à 14500 en 1993-95, soit un rythme annuel de $+1,7 \%$. Ainsi, le niveau de 2011 correspond-il à celui de 1988-89.

\section{Des variations au sein de l'espace peu dense}

La motorisation, que l'on peut observer grâce aux $E A R$, n'a pas atteint les mêmes niveaux dans les différents territoires peu denses. Le nombre de voitures par adulte est un bon indicateur de saturation si l'on prend comme horizon la situation d'une voiture par adulte, c'est-à-dire par personne en âge de conduire. Cet horizon paraît encore éloigné puisque le taux atteint dans les trois types d'espaces périurbains - les moins denses, ceux de densité intermédiaires et les plus denses - n'est encore que de 0,83 en
2011-2013. Cependant, la progression ralentit dans le périurbain le plus dense dont le taux semble devoir passer au-dessous de celui des deux autres. Il pourrait en outre être rattrapé d'ici deux ou trois ans par les espaces de très faible densité (cf. figure IV). Ainsi le processus de saturation semble engagé dans les espaces périurbains les plus denses $(\mathrm{A} 3+\mathrm{B} 3)$ là où la motorisation est la plus élevée à presque 1,7 véhicules par ménage.

L'utilisation d'une automobile varie aussi selon le type de ménage et le type d'espace peu dense. Les kilométrages les plus importants sont observés dans les contextes périurbains. Ils sont supérieurs d'environ $10 \%$ à ceux des espaces de très faible densité (A1a) et petits pôles (A1b+B1), qui sont plus isolés (cf. tableau 4). À part cette opposition, les différences sont assez faibles et rarement significatives.

Les types d'espace peu dense connaissent enfin des niveaux de chômage différents, avec des évolutions grossièrement parallèles. Pour tout l'espace peu dense, le taux d'emploi (proportion des actifs occupés sur l'ensemble des actifs) est

Figure IV

Évolution du nombre de véhicules par adulte de 19 ans et plus par type d'espace peu dense

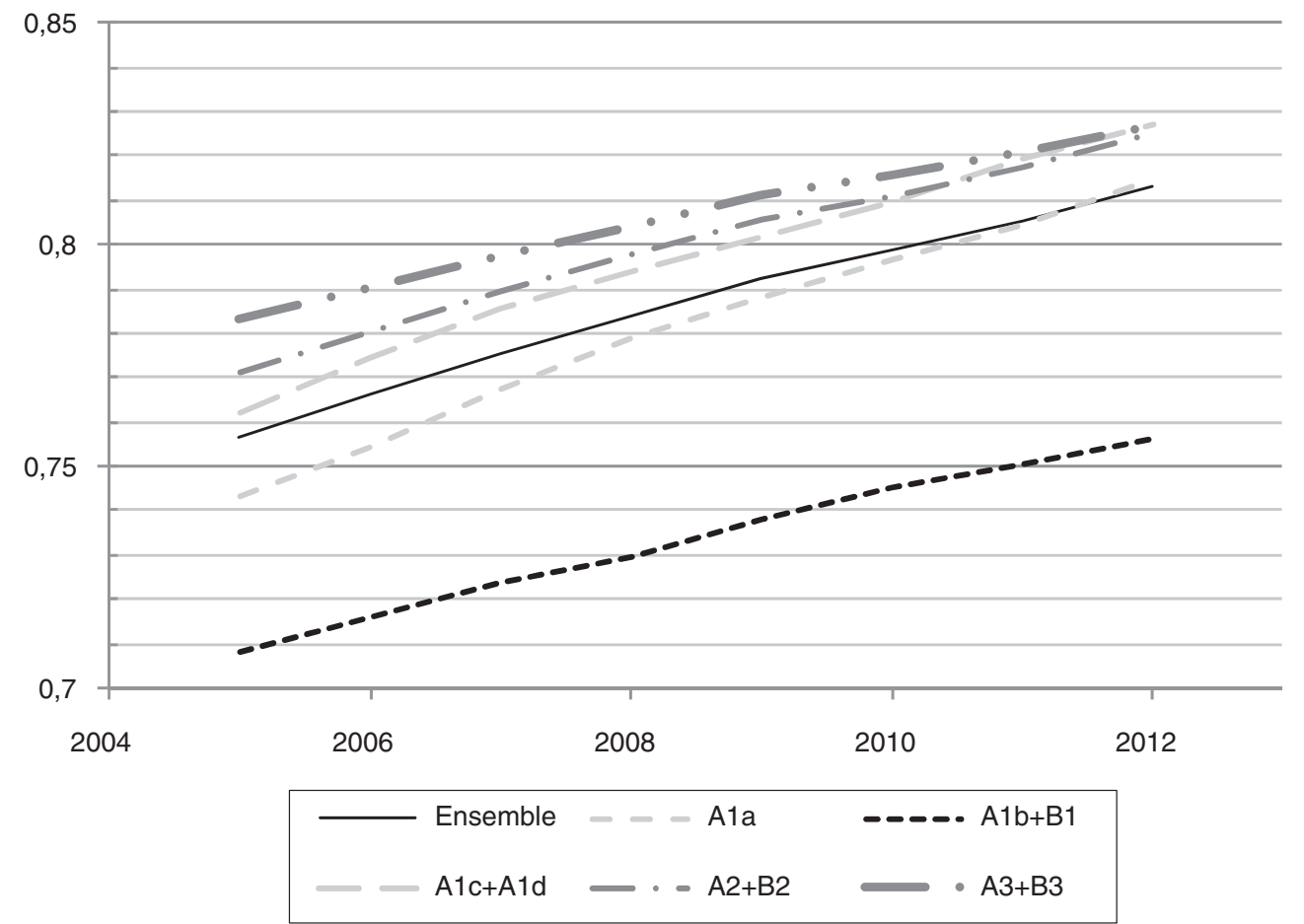

Lecture: A1a: espace de très faible densité ; $A 1 b+B 1$ : petits pôles; $A 1 c+A 1 d$ : espace à dominante périurbaine de faible densité ; $A 2+B 2$ espace à dominante périurbaine de densité intermédiaire ; $A 3+B 3$ : espace à dominante périurbaine de forte densité.

Champ : ménages qui résident dans les communes hors des unités urbaines de 10000 habitants et plus.

Source : Insee, Recensement de la population 2006 et 2011 (exploitation principale). 
maximal à 91,5\% en 2007 et 2008 et descend à 90,0 \% en 2012 (source EAR, moyenne glissante sur 3 ans). Les espaces les moins denses (A1a et A1c+A1d) sont proches de cette courbe. Mais les espaces périurbains montrent un taux supérieur, d'environ 0,5 point pour le moyennement dense $(A 2+B 2)$, et 1,5 points pour le plus dense (A3+B3). Le taux d'emploi dans les petits pôles $(\mathrm{A} 1 \mathrm{~b}+\mathrm{B} 1)$, en revanche, est d'environ 2 points au-dessous de la valeur d'ensemble avant 2007, et l'écart s'accroît à 2,5 points en 2012.

\section{La croissance de la motorisation ne compense pas la moindre utilisation des véhicules après 2008}

Dans les années 1970 et 1990, la croissance de la motorisation des ménages a conduit à une augmentation du kilométrage moyen par ménage alors que chaque voiture était moins utilisée. En est-il encore de même alors que l'augmentation de la motorisation dans l'espace peu dense est davantage portée par les ménages inactifs, dont la part dans le parc automobile est passée de 21,7 à 24,1 \% entre 2004 et 2013 ?

Pour répondre à cette question, nous simulons l'évolution du kilométrage des ménages entre 2004 et 2013, en laissant varier au cours du temps, selon les $E A R$, la répartition des ménages d'après une classification en cinq groupes dont la motorisation et l'utilisation des voitures sont bien différenciées, tout en laissant fixe au niveau de 2008 le kilométrage des voitures par groupe de ménages. La classification est construite à partir du nombre d'actifs occupés dans le ménage et de la présence ou non d'une personne de 75 ans ou plus, et les kilométrages sont fixés au niveau moyen observé pour chaque groupe lors de l'ENTD 2008 : 6500 km chez les ménages inactifs comptant au moins une personne de 75 ans ou plus, $10000 \mathrm{~km}$ chez les autres ménages inactifs, $11100 \mathrm{~km}$ chez les ménages actifs sans emploi, $13300 \mathrm{~km}$ lorsqu'il y a un emploi et $14600 \mathrm{~km}$ lorsqu'il $\mathrm{y}$ en a deux ${ }^{13}$.

Le kilométrage simulé progresse légèrement sous l'effet de la croissance de la motorisation, d'environ $1 \%$ entre 2005 et 2012. Or pendant le même temps, le kilométrage moyen par ménage a diminué de plus de $5 \%$ (cf. tableau 3). Cette forte divergence entre la simulation et l'observation d'après Parc Auto souligne la vigueur de la tendance à la baisse des kilométrages chez les habitants des zones peu denses, quels que soient leur âge et leur niveau d'activité (cf. figure V).

\section{Aller du domicile au travail : une demande ambiguë de mobilité automobile}

\section{De la distance domicile-travail à l'utilisation des voitures}

En ordre de grandeur, dans l'espace peu dense, environ 8 actifs occupés sur 10 vont travailler en voiture et la même proportion dispose d'une voiture à cette fin. L'exercice d'un emploi et la disposition d'une automobile vont de pair car l'accès à l'emploi dépend généralement de l'automobile et le coût de ces déplacements doit être compensé par les revenus du travail.

13. Source ENTD 2008, moyenne arrondie à 100 km du kilométrage annuel d'une voiture écrêté à $50000 \mathrm{~km} / \mathrm{an}$.

Tableau 4

Kilométrage annuel moyen d'un véhicule d'un ménage par types d'espace peu dense (ENTD 2008 écrêté à 50000 km par an)

\begin{tabular}{|c|c|c|c|c|c|c|}
\hline \multirow{2}{*}{$\begin{array}{l}\text { Kilométrage annuel d'un véhicule appartenant } \\
\text { à un... }\end{array}$} & \multirow{2}{*}{$\begin{array}{c}\text { Espaces de très } \\
\text { faible densité } \\
\text { A1a }\end{array}$} & \multirow{2}{*}{$\begin{array}{l}\text { Petits } \\
\text { pôles } \\
\text { A1b+B1 }\end{array}$} & \multicolumn{3}{|c|}{ Espaces à dominante périurbaine } & \multirow{2}{*}{$\begin{array}{l}\text { Espace } \\
\text { peu } \\
\text { dense }\end{array}$} \\
\hline & & & $A 1 c+A 1 d$ & $\mathrm{~A} 2+\mathrm{B} 2$ & $\mathrm{~A} 3+\mathrm{B} 3$ & \\
\hline ... ménage sans actifs occupés & 9291 & 8301 & 9588 & 8865 & 9279 & 9047 \\
\hline ... ménage avec actifs occupés & 13544 & 13522 & 14648 & 14534 & 14094 & 14205 \\
\hline Ensemble des véhicules & 12210 & 12017 & 13410 & 13290 & 13286 & 12971 \\
\hline $\begin{array}{l}\text { Part du parc de véhicules dont disposent } \\
\text { les ménages avec actifs occupés (\%) }\end{array}$ & 69 & 71 & 75 & 78 & 83 & 76 \\
\hline
\end{tabular}

Champ : ménages qui résident dans les communes hors des unités urbaines de 10000 habitants et plus.

Source: Service de l'observation et des statistiques du Ministère de l'Environnement, de l'Énergie et de la Mer (SOeS), Insee, Inrets, ENTD 2008, exploitation CASD. 
Entre les trois types d'espace peu dense, les distances moyennes sur la classe [0,80 km[ s’étagent dans le même ordre que les kilométrages annuels des voitures (cf. tableau 4 et encadré 2

Figure V

Évolution des kilométrages annuels par ménage, selon le panel Parc Auto et selon une simulation ne dépendant que de la motorisation des ménages répartis en cinq classes (base 100 en 2005)

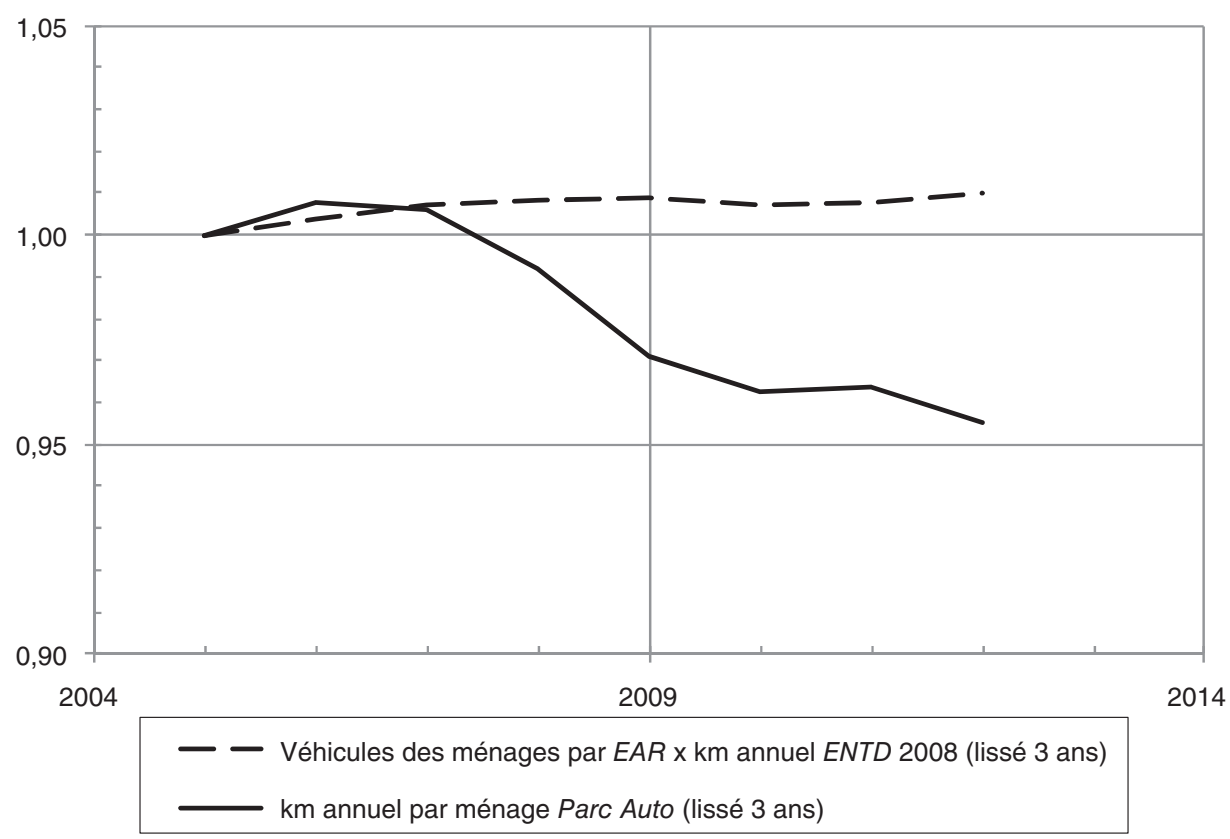

Lecture : la courbe en pointillé donne l'évolution simulée du kilométrage annuel d'un ménage s'il ne dépendait que des effectifs et de la motorisation de cinq classes de ménages constituées en fonction du nombre d'actifs employés et de la présence d'inactifs de 75 ans et plus, le kilométrage étant fixé au niveau moyen pour chaque groupe selon l'ENTD 2008. La courbe en continu donne l'évolution empirique tirée du panel Parc Auto.

Champ : ménages motorisés qui résident hors des unités urbaines de 10000 habitants et plus.

Source : TNS Sofres, panel Parc Auto (2004-2013) ; SOeS, Insee, Inrets, Enquête nationale Transports et déplacements 2008 ; Insee,

Recensement de la population 2006 et 2011 (exploitation principale).

\section{Encadré 2 \\ ANALYSER LES ÉVOLUTIONS DE LA DISTANCE DOMICILE-TRAVAIL}

La distance domicile-travail est ici calculée comme la distance à vol d'oiseau entre les chefs-lieux des communes de résidence et de travail. De manière générale, les distributions de distances de déplacements sont proches du type Log-Normale : extrêmement déséquilibrées vers les faibles valeurs, la queue de distribution pesant fortement sur la moyenne qui doit être écrêtée (Nair et Bhat, 2003). Or différentes situations de mobilité existent suivant la plage de distance. C'est pourquoi, quand l'échantillon le permet, il est intéressant d'analyser cette évolution avec plusieurs indicateurs: sur des classes, des moyennes à l'intérieur des classes, une distance ou un temps estimés sur un réseau routier, etc. Les distances les plus élevées sortent en effet du champ de la mobilité régulière, pratiquée plusieurs fois par semaine. Le calcul de la moyenne est écrêté à $80 \mathrm{~km}$ à vol d'oiseau, seuil habituellement utilisé pour séparer les mobilités dites «locales " et «à longue distance " (Armoogum et al., 2010). Dans l'intervalle [0, 80 km[, on distinguera aussi la valeur nulle qui signifie un travail dans la commune de résidence et pour laquelle l'estimation de temps de parcours ou de distance sur le réseau routier est indisponible.

L'intervalle ]0, $80 \mathrm{~km}$ [ peut ensuite être divisé en deux classes: celle des distances dont le franchissement pèse « raisonnablement » sur l'emploi du temps et le reste. Nous fixons la séparation à $30 \mathrm{~km}$, qui correspond

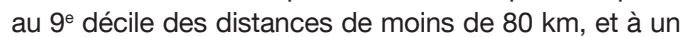
temps légèrement supérieur à 30 minutes par déplacement à une vitesse de $57 \mathrm{~km} / \mathrm{h}$. C'est la vitesse moyenne calculée sur l'ensemble des trajets domicile-travail dont nous estimons la durée et la distance routière grâce au distancier OrbisGis H2 Network (Bocher et al., 2014), selon les valeurs de vitesses sur le réseau routier IGN Route 500 en heure de pointe, données par Hilal (2008). Ce calcul ne tient pas compte d'éventuels péages routiers ni de la sinuosité des routes.

Les déplacements frontaliers qui concernent 1,6\% des actifs ne sont pas inclus dans la distance moyenne écrêtée à $80 \mathrm{~km}$, ni dans les estimations de distance ou de temps réseau. 
pour la méthode d'estimation de la distance domicile-travail) : plus élevés en périurbain, minimums dans les petits pôles et intermédiaires dans la très faible densité. Cet ordre des distances reflète principalement la part du travail dans la commune de résidence. La densité locale dans un petit pôle promet une probabilité plus importante d'y trouver un emploi (44\% en 2011) et d'échapper un peu à la dépendance automobile. Cette part est encore de $33 \%$ dans les espaces de très faible densité, à cause de la présence significative d'agriculteurs ou d'actifs travaillant à leur compte dans les villages. Elle est minimum à $20 \%$ en périurbain métropolisé de forte densité (A3+B3).

Mais entre les types de territoires périurbains, il n'y a pas de relation directe entre distance domicile-travail et kilométrage annuel. Lorsque la densité de voisinage est plus élevée, la distance domicile-travail est plus longue $(12,7 \mathrm{~km}$ en périurbain de forte densité $\mathrm{A} 3+\mathrm{B} 3$ contre 12,2 km en A1c+A1d, sur l'intervalle [0, $80 \mathrm{~km}[$ ). Pourtant le kilométrage annuel d'une voiture, d'après l'ENTD 2008, est légèrement inférieur pour les ménages avec actifs occupés (14 100 km en $\mathrm{A} 3+\mathrm{B} 3$ contre $14600 \mathrm{~km}$ en $\mathrm{A} 1 \mathrm{c}+\mathrm{A} 1 \mathrm{~d})$. Résider à proximité d'une grande agglomération peut donc avoir un double effet : certes les actifs travaillent un peu plus loin de leur domicile, mais il est possible de réduire l'usage des véhicules pour les motifs non obligés.

Entre 2006 et 2011, l'utilisation de l'automobile pour aller travailler a globalement suivi celle des distances qui augmentent sous le double effet de l'éloignement croissant entre communes de résidence et de travail (cf. tableau 5, colonne « 0 non inclus ») et de la diminution du travail dans la commune (cf. tableau 6, classe « $0 \mathrm{~km} »)$. L'allongement des distances domicile-travail renforce donc le « couplage » entre emploi et usage de l'automobile. Il a un impact sur les kilométrages parcourus par les véhicules, mais pas nécessairement de la même façon selon les territoires car les conditions de circulation y sont différentes ${ }^{14}$, ainsi que la distribution des distances à l'emploi.

14. Selon les territoires, la voiture n'est pas utilisée dans les mêmes conditions de trafic, ce qui peut influencer le comportement des actifs. Une illustration est donnée par la comparaison des estimations des temps de trajet et des distances pour les navettes hors de la commune de résidence, qui reflètent l'éloignement par rapport aux pôles d'emploi du voisinage (dans l'intervalle ]0, $80 \mathrm{~km}$ D. La distance est supérieure dans les espaces de très faible densité et les petits pôles, mais les temps de parcours y sont inférieurs à ceux des périurbains. En effet, plus les périurbains habitent près des grandes agglomérations, plus ils ont de chance d'aller y travailler en circulant dans des espaces de forte densité où l'on roule moins vite.

\section{Tableau 5}

Distances, parts modales de la voiture, temps et vitesses de déplacement en heures de pointe pour les trajets domicile-travail

\begin{tabular}{|c|c|c|c|c|c|c|c|c|c|c|c|}
\hline \multirow{3}{*}{\multicolumn{2}{|c|}{$\begin{array}{c}\text { Catégories de communes } \\
\text { dans l'espace peu dense } \\
\text { (d'après la typologie des densités } \\
\text { locale et de voisinage } \\
\text { au RP 2009) }\end{array}$}} & \multicolumn{4}{|c|}{$\begin{array}{c}\text { Distances moyennes domicile- } \\
\text { travail à vol d'oiseau et tous modes } \\
\text { de transport } \\
\text { (en km, écrêtées à } 80 \mathrm{~km} \text { ) }\end{array}$} & \multirow{2}{*}{\multicolumn{2}{|c|}{$\begin{array}{l}\text { Mode principal } \\
\text { " voiture, camion, } \\
\text { fourgonnette " } \\
(\text { en } \%)\end{array}$}} & \multirow{2}{*}{\multicolumn{2}{|c|}{$\begin{array}{l}\text { Temps moy. } \\
\text { des trajets } \\
\text { domicile-travail } \\
\text { en voiture (1) } \\
\text { (en minutes, } \\
\text { heures de pointe) } \\
\text { o non inclus }\end{array}$}} & \multirow{2}{*}{\multicolumn{2}{|c|}{$\begin{array}{l}\text { Vitesses moy. } \\
\text { des trajets } \\
\text { domicile-travail } \\
\text { en voiture (1) } \\
\text { (en } \mathrm{km} / \mathrm{h} \text {, heures } \\
\text { de pointe) } \\
0 \text { non inclus }\end{array}$}} \\
\hline & & \multicolumn{2}{|c|}{0 inclus } & \multicolumn{2}{|c|}{0 non inclus } & & & & & & \\
\hline & & 2006 & 2011 & 2006 & 2011 & 2006 & 2011 & 2006 & 2011 & 2006 & 2011 \\
\hline $\begin{array}{l}\text { Espaces de très } \\
\text { faible densité }\end{array}$ & A1a & 10,2 & 10,9 & 16,3 & 16,7 & 77,6 & 79,8 & 23,6 & 23,9 & 58,7 & 59,0 \\
\hline Petits pôles & $A 1 b+B 1$ & 8,6 & 9,4 & 16,4 & 17,0 & 76,7 & 78,2 & 26,7 & 27,2 & 54,3 & 54,9 \\
\hline \multirow{3}{*}{$\begin{array}{l}\text { Espaces à dominante } \\
\text { périurbaine }\end{array}$} & $A 1 c+A 1 d$ & 11,4 & 12,2 & 16,0 & 16,5 & 82,2 & 84,1 & 23,8 & 24,2 & 55,9 & 56,5 \\
\hline & $\mathrm{A} 2+\mathrm{B} 2$ & 12,0 & 12,6 & 16,2 & 16,6 & 83,5 & 84,6 & 26,0 & 26,3 & 53,2 & 53,7 \\
\hline & $\mathrm{A} 3+\mathrm{B} 3$ & 12,4 & 12,7 & 15,8 & 16,0 & 83,6 & 83,9 & 29,1 & 29,3 & 48,2 & 48,6 \\
\hline \multicolumn{2}{|c|}{ Ensemble de l'espace peu dense } & 11,2 & 11,9 & 16,1 & 16,5 & 81,4 & 82,9 & 25,9 & 26,2 & 53,6 & 54,1 \\
\hline \multicolumn{12}{|c|}{$\begin{array}{l}\text { 1. Les temps et vitesses des trajets domicile-travail sont calculés sur les trajets dont la distance à vol d'oiseau est inférieure à } 80 \mathrm{~km} \text {, } \\
\text { non nulle, et pour lesquels le mode principal est « voiture, camion, fourgonnette. }\end{array}$} \\
\hline \multicolumn{12}{|c|}{$\begin{array}{l}\text { Lecture : entre } 2006 \text { et } 2011 \text {, dans les espaces de très faible densité (A1a), la distance domicile-travail à moins de } 80 \mathrm{~km} \text {, incluant le } \\
\text { travail dans la commune de résidence, a augmenté de } 10,2 \mathrm{~km} \text { à 10,9 km. En excluant le travail dans la commune de résidence, les } \\
\text { valeurs sont de } 16,3 \mathrm{~km} \text { et 16,7 km. La part d'utilisation de la voiture est montée de } 77,6 \% \text { à } 79,8 \% \text { (vers toutes communes); le temps } \\
\text { moyen des trajets en voiture (hors commune de résidence), de 23,6min à 23,9min, la vitesse sur le réseau routier passant de } 58,7 \mathrm{~km} / \mathrm{h} \\
\text { à } 59,0 \mathrm{~km} / \mathrm{h} \text {. } \\
\text { Champ : actifs occupés des communes hors des unités urbaines de } 10000 \text { habitants et plus travaillant à moins de } 80 \mathrm{~km} \text { du lieu de } \\
\text { résidence (hors étranger). } \\
\text { Source : Insee, Recensement de la population } 2006 \text { et } 2011 \text { (exploitation complémentaire); distancier H2 Network sur IGN Route } 500 \text {. }\end{array}$} \\
\hline
\end{tabular}




\section{Des divergences dans l'évolution de la distribution des distances entre les territoires peu denses}

L'utilisation des voitures pour aller travailler varie selon la classe de distance où se situe la navette domicile-travail. Elle est maximum dans la classe ]0,30 km] où elle dépasse souvent $95 \%$ en 2011 (cf. tableau 6). Dans les espaces périurbains où la proportion des emplois exercés dans la commune de résidence est faible, les distances domicile-travail non nulles se situent le plus souvent dans cette classe des courtes distances (71\%), en lien avec la part modale plus importante de la voiture. L'usage de la voiture est légèrement plus faible pour les distances supérieures, dans la classe ]30, $80 \mathrm{~km}[$ : de $93 \%$ dans les espaces de très faible densité à $75 \%$ dans le périurbain métropolisé $(A 3+B 3)$ où l'offre de transport collectif existe. Dans l'ensemble, cet usage est en légère diminution (- 0,3 point) par rapport à 2006. Néanmoins, l'utilisation modérée de la voiture par ceux qui travaillent dans leur commune de résidence a augmenté de deux points dans l'ensemble de l'espace peu dense entre les RP de 2006 et 2011. L'évolution de la distribution des distances met en relief le cas des petits pôles $(\mathrm{A} 1 \mathrm{~b}+\mathrm{B} 1)$ et du périurbain le moins dense $(\mathrm{A} 1 \mathrm{C}+\mathrm{A} 1 \mathrm{~d})$ où la classe des distances élevées [30, $80 \mathrm{~km}$ [ augmente le plus (+ 0,9 point) tout en restant associée à une part modale très élevée de l'automobile.

Tableau 6

Classes de distance domicile-travail et parts du mode principal « voiture, camion, fourgonnette »: évolution 2006-2011

\begin{tabular}{|c|c|c|c|c|c|c|}
\hline \multicolumn{2}{|c|}{$\begin{array}{l}\text { Catégories de communes } \\
\text { dans l'espace peu dense } \\
\text { (selon les densités locale } \\
\text { et de voisinage au RP 2009) }\end{array}$} & $\begin{array}{l}\text { Classe de distance } \\
\text { domicile-travail } \\
\text { à vol d'oiseau (1) }\end{array}$ & $\begin{array}{l}\text { Mode principal } \\
\text { «voiture, etc. " } \\
\text { en } 2006(\%)\end{array}$ & $\begin{array}{c}\text { Part de chaque } \\
\text { classe au RP } \\
2006(\%)\end{array}$ & $\begin{array}{l}\text { Mode principal } \\
\text { «voiture, etc. " } \\
\text { en } 2011 \text { (\%) }\end{array}$ & $\begin{array}{l}\text { Part de chaque } \\
\text { classe au RP } \\
2011(\%)\end{array}$ \\
\hline \multirow{4}{*}{$\begin{array}{l}\text { Espaces de très faible } \\
\text { densité }\end{array}$} & \multirow{4}{*}{ A1a } & $0 \mathrm{~km}$ & 46,2 & 35,9 & 49,1 & 33,6 \\
\hline & & ]0, $30 \mathrm{~km}]$ & 96,3 & 52,4 & 96,7 & 54,0 \\
\hline & & ]30, $80 \mathrm{~km}[$ & 93,6 & 8,8 & 93,4 & 9,4 \\
\hline & & {$[80 \mathrm{~km}, \max ]$} & 75,5 & 2,9 & 74,9 & 3,0 \\
\hline \multirow{4}{*}{ Petits pôles } & \multirow{4}{*}{$A 1 b+B 1$} & $0 \mathrm{~km}$ & 58,5 & 47,0 & 59,6 & 44,2 \\
\hline & & ]0, $30 \mathrm{~km}]$ & 94,2 & 42,7 & 94,5 & 44,2 \\
\hline & & ]30, $80 \mathrm{~km}[$ & 89,5 & 8,1 & 89,0 & 9,0 \\
\hline & & {$[80 \mathrm{~km}, \max ]$} & 73,0 & 2,2 & 73,4 & 2,6 \\
\hline \multirow{12}{*}{$\begin{array}{l}\text { Espaces à dominante } \\
\text { périurbaine }\end{array}$} & \multirow{4}{*}{$A 1 c+A 1 d$} & $0 \mathrm{~km}$ & 50,4 & 28,0 & 53,0 & 25,7 \\
\hline & & ]0, $30 \mathrm{~km}]$ & 95,9 & 60,8 & 96,4 & 62,1 \\
\hline & & ]30, $80 \mathrm{~km}[$ & 90,6 & 8,7 & 90,2 & 9,6 \\
\hline & & {$[80 \mathrm{~km}, \max ]$} & 72,9 & 2,5 & 73,6 & 2,6 \\
\hline & \multirow{4}{*}{$\mathrm{A} 2+\mathrm{B} 2$} & $0 \mathrm{~km}$ & 54,5 & 25,3 & 56,0 & 23,6 \\
\hline & & ]0, $30 \mathrm{~km}]$ & 94,8 & 64,5 & 95,1 & 65,4 \\
\hline & & ]30, 80 km[ & 84,3 & 8,2 & 83,7 & 8,8 \\
\hline & & {$[80 \mathrm{~km}, \max ]$} & 73,0 & 2,0 & 74,2 & 2,2 \\
\hline & \multirow{4}{*}{$A 3+B 3$} & $0 \mathrm{~km}$ & 54,9 & 20,9 & 56,4 & 20,3 \\
\hline & & ]0, $30 \mathrm{~km}]$ & 92,9 & 70,5 & 92,7 & 70,7 \\
\hline & & ]30, 80 km[ & 74,7 & 6,9 & 75,1 & 7,1 \\
\hline & & [80 km, max] & 74,5 & 1,7 & 74,4 & 1,9 \\
\hline \multirow{4}{*}{\multicolumn{2}{|c|}{$\begin{array}{l}\text { Ensemble (communes hors des UU } \\
\text { de } 10000 \text { hab. et }+ \text { ) }\end{array}$}} & $0 \mathrm{~km}$ & 53,5 & 29,8 & 55,3 & 27,7 \\
\hline & & ]0, $30 \mathrm{~km}]$ & 94,7 & 59,9 & 95,0 & 61,1 \\
\hline & & ]30, 80 km[ & 86,3 & 8,1 & 86,0 & 8,8 \\
\hline & & {$[80 \mathrm{~km}, \max ]$} & 73,6 & 2,2 & 74,0 & 2,4 \\
\hline \multicolumn{7}{|c|}{$\begin{array}{l}\text { 1. - } 0 \mathrm{~km} \text { : actifs occupés qui travaillent dans leur commune de résidence. } \\
\text { - ]0,30 km] : navettes domicile-travail courtes hors de la commune de résidence mais à moins de } 30 \mathrm{~km} \text {. } \\
\text { - ]30,80 km[: navettes domicile-travail moyennes entre } 30 \text { et } 80 \mathrm{~km} \text { (bornes exclues) } \\
\text { - [80 km ; max] : longues navettes domicile-travail supérieures à } 80 \mathrm{~km}\end{array}$} \\
\hline
\end{tabular}

Champ : actifs occupés des communes hors des unités urbaines de 10000 habitants et plus (hors travail à l'étranger).

Source : Insee, Recensement de la population 2006 et 2011 (exploitation complémentaire). 


\section{L'utilisation par les actifs de leur voiture pour aller au travail prime de plus en plus sur les autres motifs}

$\mathbf{N}$ ous constatons que les actifs utilisent davantage leurs voitures pour aller travailler, à des distances croissantes qui les rendent captifs de ce mode, et, parallèlement, les ménages (actifs ou inactifs) roulent moins : il doit s'ensuivre que la part des kilomètres non dévolus au domicile-travail diminue. Pour le vérifier, il faudrait observer l'évolution récente des distances parcourues selon les motifs pour lesquels les ménages utilisent leurs voitures, mais nous ne pouvons nous appuyer que sur des jeux de données partiellement pertinents. Les enquêtes nationales sur les transports permettent de comparer tous les motifs de déplacement collectés par un carnet véhicule pendant 7 jours, seulement en 1994 et 2008 mais pas au-delà. Toutefois, il paraît logique que ce mouvement ait commencé dès le début des années 2000. Le panel Parc Auto apporte, mais seulement depuis 2009, un complément sur les kilométrages pour déplacements touristiques (vacances et weekends avec au moins une nuitée hors du domicile).
Ainsi, entre 1994 et 2008, la répartition des kilomètres parcourus par les voitures pendant une semaine selon le motif de destination a sensiblement changé chez les ménages avec au moins un actif employé (cf. tableau 7). La part des déplacements vers le lieu de travail fixe est passée de $18 \%$ à $20 \%$ du kilométrage. Le motif " accompagner ou aller chercher quelqu'un», souvent lié au précédent quand les enfants sont déposés à l’école en allant au travail, a également progressé : de $6 \%$ à $7 \%$. Inversement, les déplacements pour achats ou pour des visites diminuaient : de $14 \%$ à $12 \%$ et de $8 \%$ à $7 \%$ respectivement ${ }^{15}$. Le phénomène est sensible dans tous les sous-espaces, sauf dans les petits pôles où la baisse (- 2 pts) pourrait être compensée par la forte hausse des autres motifs professionnels (+ 3 pts).

En fin de compte, dans l'ensemble des déplacements de moins de $100 \mathrm{~km}$ faits par un véhicule d'un ménage comptant au moins un actif occupé et vivant dans l'espace peu dense, les déplacements ayant pour origine ou destination un lieu de travail fixe représentaient $85 \mathrm{~km}$ sur 244 en 1994 (35\%) et $99 \mathrm{~km}$ sur 252 (39\%) en 2008.

15. Ces deux classes diminuent également pour les véhicules des inactifs, mais au profit des loisirs.

Tableau 7

Évolution entre 1994 et 2008 de la répartition des distances parcourues localement par une voiture d'un ménage avec actif occupé, pendant une semaine, selon les motifs de destination et par type d'espace

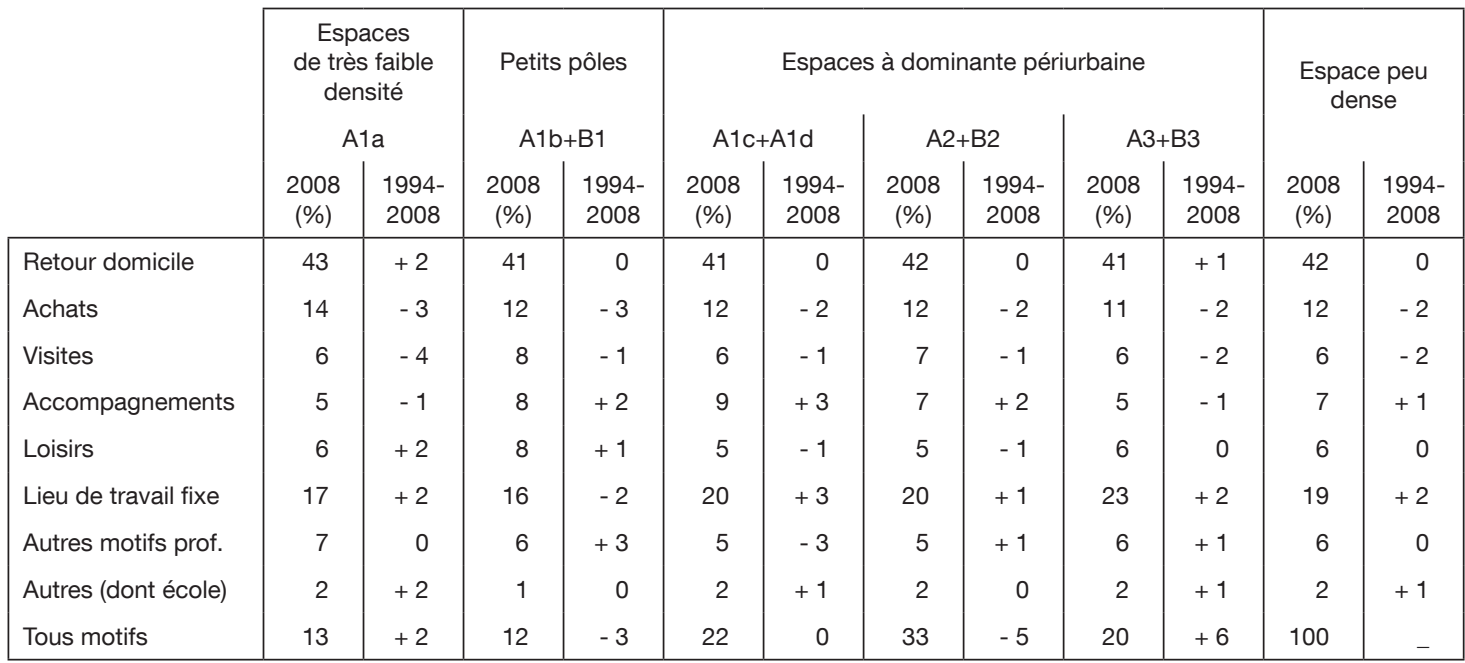

Lecture : la part des kilomètres parcourus par un véhicule pour le motif " achats " au cours d'une semaine a été de $14 \%$ en 2008 , dans les espaces de très faible densité, en baisse de - 4 points par rapport à 1994. Avec la progression du motif «lieu de travail fixe », les navettes domicile-travail comptent également davantage à l'intérieur des motifs : "retour domicile ». Dans la dernière ligne, la part des kilomètres par type d'espace.

Champ : voitures ou véhicules utilitaires légers en état de marche à disposition des ménages de l'espace peu dense comptant au moins un actif occupé, déplacements de moins de $100 \mathrm{~km}$ au compteur.

Source : SOeS, Insee, Inrets, enquête nationale Transports 1994 et enquête nationale Transports et Déplacements 2008 (exploitation CASD). 
Ce kilométrage dépassait les deux-tiers du kilométrage total observé pour $20 \%$ des véhicules en 1994, et $23 \%$ en 2008.

Selon le panel Parc Auto, le kilométrage pour le motif domicile-travail aurait, depuis lors, légèrement baissé en tendance (- $7 \mathrm{~km}$ par an sur la série 2009-2013) mais sa part continue d'augmenter dans le kilométrage total qui diminue quant à lui de $39 \mathrm{~km}$ par an. Les ménages continueraient donc à réduire leurs déplacements en voiture pour les motifs non obligés notamment les vacances et les sorties de weekend dont les kilométrages ont baissé de $25 \mathrm{~km}$ par an entre 2009 et 2013.

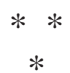

En l'absence de source statistique donnant une vision globale après 2008, la croissance de l'équipement des ménages en automobile ainsi que l'augmentation des distances domicile-travail, mises en évidence par le recensement, pourraient sembler contradictoires avec la baisse des kilométrages parcourus par les ménages, qui est observée à partir du début des années 2000 grâce au panel Parc Auto.

L'analyse distinguant les types de ménage et d'espace peu dense, et utilisant autant que faire se peut la série annuelle des EAR, montre que les facteurs traditionnellement associés à une plus grande utilisation de l'automobile ont moins d'effet qu'auparavant. Entre 2004 et 2013, la dynamique de la motorisation arrive à saturation, notamment dans la périphérie des grandes métropoles et chez les ménages avec actifs occupés. Si ces derniers tendent à utiliser plus fréquemment l'automobile pour aller travailler, c'est surtout pour les distances domicile-travail les plus courtes ou à l'intérieur de la commune de résidence. Pour les distances plus élevées, supérieures à $30 \mathrm{~km}$, la part modale de l'automobile tend à décroître légèrement. Enfin, l'augmentation du chômage à partir de 2008 fait baisser l'utilisation des automobiles, tout en maintenant le besoin d'en disposer pour répondre à une éventuelle offre d'emploi. L'exercice de simulation basé sur l'ENTD 2008 (pour les kilométrages des voitures) et les $E A R$ (pour l'évolution de la répartition de groupes de ménages aux comportements différenciés) montre cet affaiblissement des facteurs favorables à l'augmentation de la circulation. Dans ces conditions, la tendance à moins utiliser chaque voiture peut entraîner à la baisse le kilométrage des ménages. Il restait cependant à analyser les mécanismes sous-jacents à cette tendance en en cherchant les prémisses entre 1994 et 2008.

L'étude de l'utilisation des voitures des actifs occupés a montré que la part des motifs qui n’étaient pas liés au domicile-travail s'est nettement réduite entre ces deux dates. Ainsi la tendance à moins utiliser la voiture a été peu sensible à l'allongement des trajets domicile-travail. Nous pouvons alors conjecturer que les ménages avec actifs occupés ont continué à affecter de plus en plus leurs véhicules aux navettes domicile-travail et qu'ils ont recentré leurs déplacements pour d'autres motifs plus près de leur résidence, comme les autres ménages, ou de leurs lieux de travail.

\section{BIBLIOGRAPHIE}

Armoogum J., Hubert J.-P., Roux S. et Le Jeannic T. (2010), « Plus de voyages, plus de kilomètres quotidiens : une tendance à l'homogénéisation des comportements de mobilité des Français, sauf entre ville et campagne », La revue du CGDD - La mobilité des français, pp. 5-24.

Barthe L. et Milian J. (2012), « Les espaces de la faible densité - processus et scénarios », Territoires 2040, n4, pp. 151-183.

Berger M., Bouleau M et Mangeney C. (2015), «Les périurbains franciliens : vers de nouveaux comportements de mobilité ? », EchoGéo, n 34, http://echogeo.revues.org/14399.

BITRE (2012), "Traffic Growth: Modelling a Global Phenomenon », Report 128, Bureau of Infrastructure, Transport and Regional Economics, Canberra ACT.

Bocher E., Petit G. et Lecoeuvre M. (2014), «H2Network : un outil pour la modélisation et l'analyse de graphes dans le Système d'Information Géographique OrbisGIS », IFSTTAR, Rapport de recherche, https://halshs.archives-ouvertes. fr/halshs-01133333. 
Brutel C. (2014 a), Estimer les flux d'entrées sur le territoire à partir des enquêtes annuelles de recensement, Document de travail F 1403, Insee, http://www.insee.fr/fr/publications-et-services/ docs_doc_travail/F1403.pdf.

Brutel C. (2014 b), « Les immigrés récemment arrivés en France. Une immigration de plus en plus européenne ", Insee Première, $\mathrm{n}^{\circ} 1524$, novembre.

CCTN (2015), Les comptes des transports en 2014, 52 $2^{\mathrm{e}}$ rapport de la Commission des comptes des transports de la Nation, http://www.statistiques.developpement-durable.gouv.fr/fileadmin/ documents/Produits_editoriaux/Publications/ References/2015/comptes-transports-2014/rapport-comptes-transports-edition-2015-b.pdf.

Collet R., Madre J.-L. et Hivert L. (2012), «Diffusion de l'automobile en France: vers quels plafonds pour la motorisation et l'usage ? ", Économie et Statistique, n ${ }^{4}$ 457-458, pp. 123-139.

Davezies L. (2012), La crise qui vient : la nouvelle fracture territoriale, Paris, Seuil, 112 p.

Demoli Y. (2015), Automobile et stratification sociale. Diffusion, caractéristiques et coûts de l'équipement automobile en France depuis les années 1980, Thèse de doctorat de l'Institut d'études politiques de Paris.

De Solere (dir.) (2012), La mobilité urbaine en France: enseignements des années 2000-2010, Édition du CERTU, collection Références $n^{\circ} 117$, Lyon.

Floch J.-M. et Lévy D. (2011), « Le nouveau zonage en aires urbaines de 2010. Poursuite de la périurbanisation et croissance des grandes aires urbaines ", Insee Première, n ${ }^{1375}$, octobre.

François D. (2010), "Se rendre au travail : distances et temps de transport s'allongent », La revue du CGDD - La mobilité des français, pp. 83-98.

Grimal R. (2010), « Mobilité à longue distance : plus de voyages s'effectuent en train, mais les seniors restent adeptes de la voiture », La revue du CGDD - La mobilité des français, pp. 123-150.

Grimal R., Colet R. et Madre J.-L. (2013), « Is the Stagnation of Individual Car Travel a General Phenomenon in France? A Time-Series Analysis by Zone of Residence and Standard of Living ", Transport Reviews, vol. 33, n³, pp. 291-309.
Goodwin Ph. (2012), " Peak travel, peak car and the future of mobility: Evidence, unresolved issues, policy implications, and a research agenda », Discussion Paper 2012, n 13 , OECD/ ITF Roundtable on Long-run Trends in Travel Demand.

Hilal M. (2008), ODOMATRIX, Documentation, INRA, UMR1041 CESAER, 7 p., http://www2. dijon.inra.fr/cesaer/fichiers/pagesperso/hilal/ ODOMATRIX\%20ss\%20CLUF.pdf.

Hilal M., Barczak A., Tourneux F.-P., Schaeffer Y., Houdart M. et Cremer-Schulte D. (2012), Typologie des campagnes françaises et des espaces à enjeux spécifiques, Travaux en ligne, $n^{\circ} 12$, Datar, Paris.

Hubert J.-P. (2009), « Dans les agglomérations, la mobilité quotidienne des habitants diminue, et elle augmente ailleurs », Insee Première, n 1252 , juillet.

Hubert J.-P. et Delisle F. (2010), « L'allongement des déplacements quotidiens contribue à l'émergence d'espaces urbains multipolaires, tandis que la mobilité baisse au centre des grandes agglomérations ", La revue du CGDD - La mobilité des français, décembre 2010, pp. 41-56.

Hurez C. et Tebar M. (2015), «La mobilité des habitants de Rhône-Alpes : construction d'un nouveau protocole d'enquête ", dans Armoogum J., Guilloux T. et Richer C. (dir.), Mobilité en transitions. Connaître, comprendre et représenter, Cerema, collection « Rapports », Lyon, pp. 51-63.

Insee (2005), « Le nouveau mode de recensement : élaboration à travers les tests » in Godinot A. (dir.), Pour comprendre le recensement de la population, Insee Méthodes, numéro hors série.

Join-Lambert E. et Thélot C. (1981), «Les ménages roulent plus, les voitures roulent moins ». Économie et Statistique, n ${ }^{\circ}$ 136, septembre 1981, pp. 43-51.

Kuhnimof T., Armoogum J., Buehle, R., Dargay J., Martin Denstadli J. et Yamamoto T. (2012), "Men Shape a Downward Trend in Car Use among Young Adults - Evidence from six industrialized countries", Transport Reviews, vol. 32, n6, pp. 761-779.

Le Breton E. (2008), Domicile-travail. Les salariés à bout de souffle, Paris, Scrineo, collection Les carnets de l’info. 
Lipatz J.-L. (2011), "Les carroyages: des zonages pour tuer les zonages », Séminaire Belgrand, 23 juin, http://belgrand-gebd.ifsttar.fr/ fileadmin/seminaire/_2/Belgrand_23_juin_2011_ Lipatz_carroyage .pdf.

Madre J.-L. et Bussière Y. (dir.) (2002), Démographie et Transport: Villes $d u$ Nord et Villes $d u$ Sud, l’Harmattan, Paris.

Madre J.-L., Hubert J.-P. et Pistre P. (2015), «Peut-on tirer des informations conjoncturelles du recensement rénové de la population à partir des EAR ? L'exemple de la motorisation des ménages et des mobilités domicile-travail dans les commune de moins de 10000 habitants ", Actes des Journées de Méthodologie Statistique, 31 mars2 avril, http://jms.insee.fr/files/documents/ 2015/ S18_3_ACTE_V2_MADRE_JMS2015.PDF.

Metz D. (2012), « Demographic determinants of daily travel demand », Transport Policy, vol. 21, pp. 20-25.
Nair H. S. et Bhat C. R. (2003), « Modelling Trip Duration for Mobile Source Emissions Forecasting », Journal of Transportation and Statistics, vol. $6, n^{\circ} 1$, pp. 17-32.

Orfeuil J.-P. (2013), « Quand la voiture devient contrainte. ", Revue Projet, n 334, mars, p. 50-58.

Ortar N. (2009), « Entre choix de vie et gestion des contraintes : télétravailler à la campagne », Flux, nº 78, pp. 49-57.

Paul-Dubois-Taine O. (dir.) (2012), Les nouvelles mobilités dans les territoires périurbaines et ruraux, Rapport pour le Centre d'Analyse Stratégique (CAS), $n^{\circ} 47$.

Raoul E. et Casteigts M. (2011), La mobilité et les transports dans les territoires ruraux, Rapport pour le Ministère de l'Intérieur, de l'Espace rural et de l'Aménagement du Territoire et le Secrétariat d'État chargé des transports, Paris, La documentation Française. 


\section{ANNEXE 1 \\ TYPOLOGIE DES COMMUNES FRANCAISES MÉTROPOLITAINES SELON LES DENSITÉS DE POPULATION LOCALE ET DE VOISINAGE}

Deux objectifs principaux ont guidé la réalisation d'une typologie générale des communes françaises, qui sert ensuite de base pour distinguer trois configurations majeures de l'espace peu dense

1) Proposer une classification de l'ensemble des communes, à partir de critères harmonisés, simples, reproductibles dans le temps et symétriques quant à l'identification des territoires plutôt urbains ou ruraux, c'est-à-dire notamment sans désigner a priori des pôles urbains ;

2) Privilégier une approche morphologique par la densité de population en tenant compte de deux échelles spatiales: une première purement locale et une deuxième liée au voisinage des communes. Le croisement de ces deux densités permet de distinguer des classes d'espace bien plus variées qu'à partir de la seule densité locale: par exemple, des communes peu denses dans un voisinage dense qui correspondent majoritairement à des configurations périurbaines.

Étape 1 : calcul de deux niveaux de densité

autour des chefs lieux des communes

À partir des données carroyées d'un kilomètre de côté tirées du RP 2009 (Lipatz, 2011), la densité moyenne de population est calculée dans deux cercles de 2,5 km et $25 \mathrm{~km}$ de rayon autour de chaque chef lieu de commune. Les résultats obtenus sont affectés aux communes correspondantes.
L'utilisation de données carroyées permet en particulier de se libérer de l'irrégularité du maillage communal qui biaise sensiblement les variations de densité. Par ailleurs, le choix des tailles de cercles découle de constatations empiriques simples quant aux comportements des individus : $2,5 \mathrm{~km}$ de rayon correspond environ à la distance parcourue à pied par un individu en 30 minutes et $25 \mathrm{~km}$ par la distance moyenne parcourue en voiture durant le même laps de temps. En outre, $15,7 \mathrm{~km}^{2}$ (superficie d'un cercle de $2,5 \mathrm{~km}$ de rayon) correspond environ à la taille moyenne des communes françaises métropolitaines, et un cercle de $25 \mathrm{~km}$ de rayon peut représenter la superficie dans laquelle sont réalisées les activités quotidiennes.

Étape 2 : définition de classes de communes à partir des déciles et quartiles de densité locale et de voisinage

Deux densités ayant été calculées pour chaque commune, la procédure de classification consiste ensuite à croiser les densités de population locale et de voisinage à partir des distributions des déciles (D) et des quartiles (Q) des populations résidentes (cf. figure). 12 classes de communes ont été retenues en donnant la primeur, par un découpage plus fin, aux classes de communes de faible et de moyenne densité. Le codage des classes de communes est effectué comme suit : de $A$ à $C$ selon trois niveaux de densité locale (le quartile $Q 1$ séparant $A$ et $B$, et la médiane $Q 2$ séparant $B$ et $\mathrm{C}$ ) et de 1 à 3 pour leurs équivalents en densité de

Figure

Classes de communes définies par croisement des densités de population locale et de voisinage à partir des distributions des déciles et des quartiles des populations résidentes

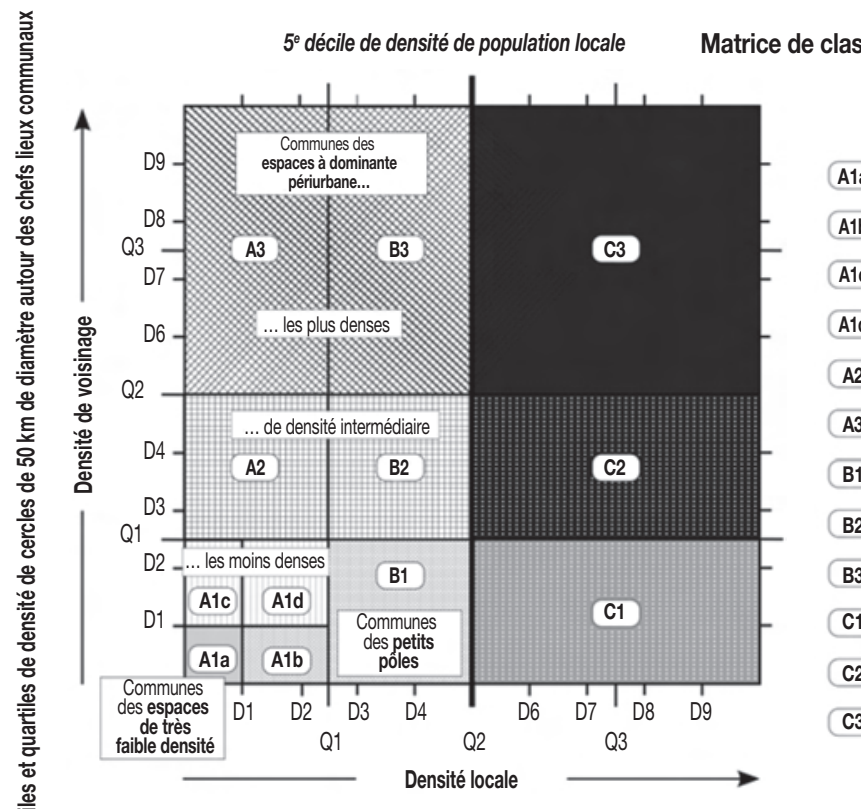

Déciles et quartiles de densité de cercles de $5 \mathrm{~km}$ de diamètre autour des chefs lieux communaux
A1a Communes trés trés peu denses

A1b Communes peu denses dans un voisinage trés peu dense

A1c Communes trés peu denses dans un voisinage peu dense

A1d Communes peu denses dans un voisinage peu dense

A2 Communes peu denses dans un voisinage presque dense

A3 Communes peu denses dans un voisinage dense et plus

B1 Communes presque denses dans un voisinage peu dense

B2 Communes presque denses dans un voisinage presque dense

B3 Communes presque denses dans un voisinage dense et plus

C1 Communes denses et plus dans un voisinage peu dense

C2 Communes denses et plus dans un voisinage presque dense

C3 Communes denses et plus dans un voisinage dense et plus

Source : Insee, Recensement de la population 2009 (données carroyées $-1 \mathrm{~km}$ de côté); IGN, GEOFLA édition 2013 
voisinage dans chaque classe de densité locale. En outre, quatre classes sont détaillées pour les espaces les moins denses (A1) : $\mathrm{A} 1 \mathrm{a}=$ croisement des premiers déciles soit $[0, D 1] ; A 1 b=[D 1, Q 1[$ en densité locale et $[0, \mathrm{D} 1]$ en densité de voisinage ; $\mathrm{A} 1 \mathrm{c}=[0, \mathrm{D} 1]$ en densité locale et [D1,Q1[ en densité de voisinage; $\mathrm{A} 1 \mathrm{~d}=$ [D1,Q1[ dans les deux densités.

Étape 3 : trois figures de communes dans l'espace peu dense selon les densités locales et de voisinage

À partir de ces 12 classes de communes, la typologie permet de distinguer trois catégories principales de communes peu denses: celles qui composent les espaces de très faible densité (A1a : faibles densités locales et de voisinage), les petits pôles (A1b+B1 : plus fortes densités locales) et les espaces à dominante périurbaine $(A 1 C+A 1 d, A 2+B 2, A 3+B 3$ : de densités de voisinage croissantes). Leurs assises spatiales en France métropolitaine sont très différentes, mais elles sont toutes les trois composées essentiellement de communes hors des unités urbaines de 10000 habitants et plus : de $70,6 \%$ en $\mathrm{A} 3$ + B3 à 99,9\% en A1a (cf. tableau ci-dessous et carte du texte).
En définitive, par rapport à la typologie Insee 2010 des aires urbaines :

- les espaces de très faible densité se composent exclusivement de communes de moins de 2000 habitants non agglomérées dont la population se répartit entre les communes multipolarisées $(32,6 \%)$ ou les communes isolées hors influence des pôles $(46,4 \%)$;

- les petits pôles se composent aussi d'une majorité de communes hors des unités urbaines $(60,1 \%$ des communes). 39,9\% de leur population appartient aux aires urbaines des petits pôles, le reste se répartit entre les couronnes des grands pôles $(15,0 \%)$, les autres communes multipolarisées $(11,0 \%)$ et les communes isolées hors influence des pôles $(19,6 \%)$;

- les espaces à dominante périurbaine se déclinent enfin en trois sous-catégories : des moins denses $(\mathrm{A} 1 \mathrm{C}+\mathrm{A} 1 \mathrm{~d})$ aux plus denses en voisinage (A3+B3). Toutes les trois se composent surtout de communes hors des unités urbaines (de $75 \%$ à 96,7\% des communes) et leur population appartient majoritairement aux couronnes des grands pôles pour les types A2+B2 $(60,1 \%)$ et A3+B3 (83,3 \%). La population du type A1C+A1d se partage entre les couronnes des grands pôles $(38,9 \%)$ et les communes multipolarisées (42,6\%).

Tableau

Croisement de la typologie des communes selon les densités de population locale et de voisinage avec le zonage Insee des aires urbaines $\mathbf{2 0 1 0}$ pour les communes de l'espace peu dense

\begin{tabular}{|c|c|c|c|c|c|c|c|}
\hline \multirow{2}{*}{ Zonage en aires urbaines 2010} & \multicolumn{7}{|c|}{$\begin{array}{l}\text { Typologie des communes françaises selon les densités locales et de voisinage } \\
\text { (d'après RP 2009) }\end{array}$} \\
\hline & & A1a & $A 1 b+B 1$ & $\begin{array}{l}\text { A1c }+ \\
\text { A1d }\end{array}$ & $\mathrm{A} 2+\mathrm{B} 2$ & $A 3+B 3$ & C \\
\hline \multirow{3}{*}{ [111] Grands pôles (10 000 emplois et plus) } & Nbr_C* & 0 & 0 & 0 & 0 & 1 & 0 \\
\hline & Nbr_P* & 0 & 0 & 0 & 0 & 2688 & 0 \\
\hline & $\%$ P* & 0,0 & 0,0 & 0,0 & 0,0 & 0,1 & 0,0 \\
\hline \multirow{3}{*}{ [112] Couronnes des grands pôles } & Nbr_C* & 1021 & 386 & 3271 & 5041 & 2179 & 20 \\
\hline & $\mathrm{Nbr} \_\mathrm{P}^{*}$ & 310144 & 543896 & 1763601 & 4484891 & 2971987 & 29575 \\
\hline & $\%$ \& $P^{*}$ & 11,0 & 15,0 & 38,9 & 60,1 & 83,3 & 100,0 \\
\hline \multirow{3}{*}{$\begin{array}{l}\text { [120] Communes multipolarisées } \\
\text { des grandes AU }\end{array}$} & Nbr_C* & 408 & 115 & 1385 & 1498 & 428 & 0 \\
\hline & Nbr_P* & 106696 & 219165 & 689427 & 1331979 & 464778 & 0 \\
\hline & $\%$ \& $P^{*}$ & 3,8 & 6,0 & 15,2 & 17,8 & 13,0 & 0,0 \\
\hline \multirow{3}{*}{ [211] Moyens pôles (5 000 -10 000 emplois) } & Nbr_C ${ }^{*}$ & 2 & 37 & 2 & 14 & 2 & 0 \\
\hline & Nbr_P* & 1589 & 187227 & 3986 & 37413 & 7272 & 0 \\
\hline & $\%$ \% $P^{*}$ & 0,1 & 5,2 & 0,1 & 0,5 & 0,2 & 0,0 \\
\hline \multirow{3}{*}{ [212] Couronnes des moyens pôles } & Nbr_C* & 436 & 105 & 183 & 78 & 1 & 0 \\
\hline & Nbr_P* & 154099 & 83850 & 78974 & 35461 & 493 & 0 \\
\hline & \%_P* & 5,5 & 2,3 & 1,7 & 0,5 & 0,0 & 0,0 \\
\hline \multirow{3}{*}{ [221] Petits pôles (1 500 -5 000 emplois) } & Nbr_C* & 28 & 508 & 93 & 147 & 13 & 0 \\
\hline & $\mathrm{Nbr} \mathrm{P}^{*}$ & 10555 & 1450586 & 114448 & 450932 & 47113 & 0 \\
\hline & \%_P* & 0,4 & 39,9 & 2,5 & 6,0 & 1,3 & 0,0 \\
\hline \multirow{3}{*}{ [222] Couronnes des petits pôles } & Nbr_C* & 432 & 90 & 58 & 7 & 0 & 0 \\
\hline & Nbr_P* & 114398 & 35288 & 17469 & 1350 & 0 & 0 \\
\hline & $\% P^{*}$ & 4,1 & 1,0 & 0,4 & 0,0 & 0,0 & 0,0 \\
\hline \multirow{3}{*}{ [300] Autres communes multipolarisées } & Nbr_C* & 2969 & 406 & 2491 & 1113 & 49 & 0 \\
\hline & Nbr_P* & 809129 & 400538 & 1245073 & 840246 & 52786 & 0 \\
\hline & $\% \_P^{*}$ & 28,8 & 11,0 & 27,4 & 11,3 & 1,5 & $0,0 \rightarrow$ \\
\hline
\end{tabular}


Tableau (suite)

\begin{tabular}{|c|c|c|c|c|c|c|c|}
\hline \multirow[b]{2}{*}{ Zonage en aires urbaines 2010} & \multicolumn{7}{|c|}{$\begin{array}{l}\text { Typologie des communes françaises selon les densités locales et de voisinage } \\
\text { (d'après RP 2009) }\end{array}$} \\
\hline & & A1a & $A 1 b+B 1$ & $\begin{array}{l}\text { A1c }+ \\
\text { A1d }\end{array}$ & $\mathrm{A} 2+\mathrm{B} 2$ & $A 3+B 3$ & C \\
\hline \multirow{3}{*}{$\begin{array}{l}\text { [400] Communes isolées hors influence } \\
\text { des pôles }\end{array}$} & Nbr_C* & 5113 & 576 & 1317 & 352 & 27 & 0 \\
\hline & Nbr_P* & 1303636 & 714084 & 624660 & 281157 & 18828 & 0 \\
\hline & \%_P* & 46,4 & 19,6 & 13,8 & 3,8 & 0,5 & 0,0 \\
\hline \multirow{3}{*}{ Ensemble de l'espace peu dense } & Nbr_C* & 10409 & 2223 & 8800 & 8250 & 2700 & 20 \\
\hline & Nbr_P* & 2810246 & 3634634 & 4537638 & 7463429 & 3565945 & 29575 \\
\hline & \%_P $P^{*}$ & 100,0 & 100,0 & 100,0 & 100,0 & 100,0 & 100,0 \\
\hline \multirow{2}{*}{ Ensemble des communes } & $\%$ C2 ${ }^{* *}$ & 99,9 & 80,1 & 97,6 & 90,0 & 70,6 & 1,5 \\
\hline & $\% \_P^{\star \star}$ & 99,8 & 65 & 94,4 & 73,0 & 45,7 & 0,1 \\
\hline
\end{tabular}

* Indicateurs pour les seules communes de l'espace peu dense :

Nbr_C : nombre de communes ;

Nbr_P : somme des populations municipales au recensement 2009 ;

\%_P : proportion de la population dans chaque classe (dont $\square$ de 20 à $50 \%$

de 50 à $83,3 \%)$.

** Indicateurs par rapport à l'ensemble des communes françaises métropolitaines :

$\%$ C2 : proportion de communes de l'espace peu dense

$\%$ _P : proportion de la population au recensement 2009 qui réside dans l'espace peu dense

Lecture : en colonne, aucune commune du type A1 n'appartient à la catégorie 111 du zonage en aire urbaine de 2010 ; 1021 communes appartiennent à la catégorie 112, comptant pour 310144 personnes, $11 \%$ de la population du type A1, etc. En dernière ligne, 99,9\% des communes du type $A 1$ et $99,8 \%$ de leur population sont dans le champ de l'espace peu dense, contre 70,6 \% des communes et

$45,7 \%$ de la population pour le type $A 3+B 3$.

Champ : communes hors des agglomérations de 10000 habitants et plus.

Source : Insee, Recensement de la population 2009 (exploitation principale). 


\section{ANNEXE 2}

\section{CONSTRUIRE DES SÉRIES ANNUELLES À PARTIR DES EAR POUR UNE ANALYSE CONJONCTURELLE SUR LES COMMUNES DE MOINS DE 10000 HABITANTS}

Pour les communes de moins de 10000 habitants, les fichiers détail du RP d'une année N sont désormais composés de cinq échantillons de communes recensées de façon exhaustive lors de cinq campagnes successives de recensement, de l'année $\mathrm{N}-2$ à l'année $\mathrm{N}+2$. Chaque EAR produit donc un échantillon au cinquième de la population de ces communes, pour l'exploitation principale (fichiers LOGEMT qui informent sur la motorisation des ménages), et au vingtième ${ }^{1}$ pour l'exploitation complémentaire, (fichiers MOBPRO qui renseignent sur les mobilités domicile-travail des actifs occupés).

Construction des séries annuelles brutes à partir des Enquêtes Annuelles de Recensement (EAR)

La composition des échantillons de communes de moins de 10000 habitants, annuellement recensés, étant connue, nous pouvons extraire de chaque recensement général, depuis le RP 2006, les ménages (fichiers LOGEMT) ou les actifs occupés (fichiers MOBPRO) d'une EAR donnée. La collecte étant rendue homogène dans la série des EAR, les poids des communes le sont également. Pour l'exploitation complémentaire, ces poids valent 4 partout en France métropolitaine, sauf en Corse où ils valent 1 . Soulignons tout de même que quelques communes ont des poids non entiers si une fusion est intervenue avec une commune d'un autre groupe ou si la commune a passé le seuil des 10000 habitants entre deux EAR, dans un sens ou dans l'autre. Ces communes sont retirées des échantillons d'étude. En définitive, pour les seules communes de l'espace peu dense, les effectifs de chaque EAR sont d'environ 1700000 ménages (exploitation principale) et 460000 actifs occupés (exploitation complémentaire).

Effets de cycle des séries annuelles brutes, méthodes correctives et variations quinquennales

La composition des cinq groupes de rotation a été définie par l'Insee de manière à assurer un même effectif et une même structure démographique à chaque EAR.
Les groupes ont été équilibrés sur la base des données du recensement 1999 et d'un ensemble de variables liées aux logements, à l'âge et au sexe des individus, et ce à l'échelle régionale (Insee, 2005).

Néanmoins, l'équilibre peut poser problème lorsque l'on travaille sur des sous-échantillons de populations ou de communes. Ils sont en effet souvent moins équilibrés que l'ensemble, dès lors qu'on retire des groupes de rotation les communes intégrées aux unités urbaines de 10000 habitants et plus. Nous avons donc vérifié la stabilité du champ des communes hors des unités urbaines de 10000 habitants et plus, et de ses subdivisions par types d'espace peu dense, à l'intérieur de l'ensemble des communes de moins de 10000 habitants, en effectifs de résidences principales (ou ménage ordinaires) au cours de la période 2004-2013 (cf. tableau). Si les proportions d'ensemble sont relativement bien conservées pour un même groupe de rotation, les types $A 1 b+B 1$ (petits pôles) et A3+B3 (périurbain dense) peuvent poser problème. Ils sont en effet composés de peu de communes, plutôt peuplées, et la répartition de ces communes entre notre champ d'étude et le reste du territoire est plus variable d'un groupe de rotation à l'autre.

Plusieurs méthodes sont utilisées pour limiter ces effets de cycle : «baser » certaines séries sur les évolutions observées depuis 1999 pour le groupe de rotation interrogé l'année n (la méthode n'est pas utilisée ici) ou appliquer des moyennes mobiles sur des intervalles inférieurs à 5 ans pour lisser les niveaux (Madre et al., 2015), ce qui est retenu ici pour l'analyse de certaines séries en niveau (taux de motorisation notamment). Pour l'analyse des évolutions, la méthode retenue est celle du calcul de variation quinquennale par groupe de rotation (à composition communale identique) en évitant de décomposer selon les types d'espace (cf. tableau).

1. Le lieu de travail n'étant codé que pour un bulletin individuel sur quatre.

Tableau

Part des effectifs non pondérés de résidences principales de l'espace peu dense dans l'ensemble des communes de moins de 10000 habitants, par types d'espace et groupes de rotation, entre deux EAR

\begin{tabular}{|c|c|c|c|c|c|c|c|c|}
\hline & & $\mathrm{A} 1 \mathrm{a}$ & $A 1 b+B 1$ & $\mathrm{~A} 1 \mathrm{c}+\mathrm{A} 1 \mathrm{~d}$ & $\mathrm{~A} 2+\mathrm{B} 2$ & $\mathrm{~A} 3+\mathrm{B} 3$ & C & Total \\
\hline \multirow[t]{3}{*}{ Groupe 1} & EAR 2004 & 99,8 & 80,0 & 94,3 & 74,9 & 47,0 & 0,7 & 67,5 \\
\hline & EAR 2009 & 99,7 & 79,6 & 94,1 & 75,1 & 47,7 & 0,8 & 67,6 \\
\hline & Écart (points) & 0,0 & $-0,4$ & $-0,1$ & 0,2 & 0,7 & 0,1 & 0,2 \\
\hline \multirow[t]{3}{*}{ Groupe 2} & EAR 2005 & 99,8 & 73,2 & 95,4 & 74,5 & 50,4 & 0,3 & 67,2 \\
\hline & EAR 2010 & 99,8 & 73,1 & 95,3 & 74,8 & 50,9 & 0,3 & 67,3 \\
\hline & Écart (points) & 0,0 & $-0,1$ & 0,0 & 0,3 & 0,4 & 0,0 & 0,2 \\
\hline \multirow[t]{3}{*}{ Groupe 3} & EAR 2006 & 100,0 & 73,4 & 93,6 & 78,1 & 47,3 & 1,2 & 67,3 \\
\hline & EAR 2011 & 100,0 & 73,2 & 93,6 & 78,2 & 48,0 & 1,2 & 67,5 \\
\hline & Écart (points) & 0,0 & $-0,2$ & 0,0 & 0,0 & 0,7 & 0,0 & $0,2 \rightarrow$ \\
\hline
\end{tabular}


Tableau (suite)

\begin{tabular}{|llrrrrrrrr|}
\hline & & A1a & A1b+B1 & A1c+A1d & A2+B2 & A3+B3 & C & Total \\
\hline Groupe 4 & EAR 2007 & 99,8 & 75,2 & 94,5 & 75,6 & 49,3 & 0,6 & 68,2 \\
& EAR 2012 & 99,7 & 74,3 & 94,5 & 76,0 & 49,7 & 0,5 & 68,2 \\
& Écart (points) & 0,0 & $-0,9$ & 0,0 & 0,5 & 0,4 & 0,0 & $-0,1$ \\
\hline Groupe 5 & EAR 2008 & 100,0 & 70,3 & 94,3 & 74,6 & 49,6 & 1,3 & 66,9 \\
& EAR 2013 & 100,0 & 69,2 & 94,3 & 75,0 & 50,8 & 1,5 & 67,1 \\
& Écart (points) & 0,0 & $-1,1$ & 0,0 & 0,4 & 1,3 & 0,1 & 0,2 \\
\hline Ensemble & EAR 2004-2008 & 99,9 & 74,3 & 94,4 & 75,5 & 48,7 & 0,8 & 67,4 \\
& EAR 2009-2013 & 99,8 & 73,7 & 94,4 & 75,8 & 49,4 & 0,9 & 67,5 \\
& Écart (points) & 0,0 & $-0,6$ & 0,0 & 0,3 & 0,7 & 0,1 & 0,1 \\
\hline
\end{tabular}

Lecture : le groupe de rotation $n^{\circ} 1$ a été recensé par les EAR de 2004 et 2009. En 2009, 79,6\% des résidences principales des communes de moins de 10000 habitants du type $A 1 b+B 1$ étaient dans le champ de l'espace peu dense (c'est-à-dire : hors des agglomérations de 10000 habs et plus). Cette proportion a baissé de 0,4 point par rapport à l'EAR 2004. En gris, les écarts supérieurs à 1 point en valeur absolue.

Champ : résidences principales des communes de moins de 10000 habitants recensées par les EAR de 2004 à 2013.

Source : Insee, Recensement de la population 2006 et 2011 (exploitation principale). 
ANNEXE 3

\section{JEUX DE DONNÉES UTILISÉS}

Recensement de la population 1999: Fichier détail "Individus » au $1 / 20^{\circ}$, Insee [producteur], Centre Maurice Halbwachs $(\mathrm{CMH})$ [diffuseur].

Recensement de la population 2006-2011 Insee [producteur et diffuseur]

Fichier logements complets: fichiers FD_ LOGEMT_2006.TXT à FD_LOGEMT_2011.TXT,

Fichier Mobilités professionnelles des actifs occupés : déplacements commune de résidence / commune de travail: fichiers FD_MOBPRO_2006.TXT à FD MOBPRO_2011.TXT,

Fichiers de documentation géographique 2006 et 2012 : Tab_geo_RP2006.xls, table-appartenance-geocommunes-au-01-01-2012.xls

Données carroyées à 1 kilomètre. Carte des estimations carroyées de la population. Fichier ECP1KM_09_MET. ZIP, Insee [producteur et diffuseur].
Répertoire géographique des communes $R G C^{\circledR}$. Editions 1990 et 2012, IGN [producteur et diffuseur].

Route 500 (. Edition 2012, IGN [producteur et diffuseur].

Enquête nationale transports 1994 MEDDE SES, Insee, Inrets [producteurs] Centre Maurice Halbwachs (CMH) [diffuseur].

Enquête nationale transports et déplacements 2008 MEDDE SOeS, Insee, Inrets [producteurs] Centre Maurice Halbwachs $(\mathrm{CMH})$ [diffuseur].

Panel Parc Auto 1994-2013, TNS Sofres [producteur], IFSTTAR-AME-DEST [dépositaire].

Les calculs de distance réseau et de temps de parcours du distancier $\mathrm{H} 2$ Network sur les domicile-travail du recensement ainsi que la composition de la typologie communale des densités locale et de voisinage sont disponibles auprès des auteurs. 
